ARTICLE

\title{
Synthetic microbial communities of heterotrophs and phototrophs facilitate sustainable growth
}

\author{
Cristal Zuñiga ${ }^{1}$, Tingting Li ${ }^{2,3}$, Michael T. Guarnieri ${ }^{4}$, Jackson P. Jenkins ${ }^{2}$, Chien-Ting $\mathrm{Li}^{2}$, Kerem Bingol ${ }^{5}$,
} Young-Mo Kim (D) ${ }^{5}$, Michael J. Betenbaugh ${ }^{2} \&$ Karsten Zengler (1D) 1,6,7凶

Microbial communities comprised of phototrophs and heterotrophs hold great promise for sustainable biotechnology. Successful application of these communities relies on the selection of appropriate partners. Here we construct four community metabolic models to guide strain selection, pairing phototrophic, sucrose-secreting Synechococcus elongatus with heterotrophic Escherichia coli K-12, Escherichia coli W, Yarrowia lipolytica, or Bacillus subtilis. Model simulations reveae metabolic exchanges that sustain the heterotrophs in minimal media devoid of any organic carbon source, pointing to $S$. elongatus-E. coli K-12 as the most active community. Experimental validation of flux predictions for this pair confirms metabolic interactions and potential production capabilities. Synthetic communities bypass memberspecific metabolic bottlenecks (e.g. histidine- and transport-related reactions) and compensate for lethal genetic traits, achieving up to $27 \%$ recovery from lethal knockouts. The study provides a robust modelling framework for the rational design of synthetic communities with optimized growth sustainability using phototrophic partners.

\footnotetext{
${ }^{1}$ Department of Pediatrics, University of California, San Diego, 9500 Gilman Drive, La Jolla, CA 92093-0760, USA. ${ }^{2}$ Department of Chemical and Biomolecular Engineering, The Johns Hopkins University, 3400 North Charles Street, Baltimore, MD 21218, USA. ${ }^{3}$ Department of Immunology, School of Basic Medical Sciences, Lanzhou University, 730000 Lanzhou, China. ${ }^{4}$ National Bioenergy Center, National Renewable Energy Laboratory, Golden, CO 80401, USA. ${ }^{5}$ Earth and Biological Sciences Directorate, Pacific Northwest National Laboratory, Richland, WA 99354, USA. ${ }^{6}$ Center for Microbiome Innovation, University of California, San Diego, 9500 Gilman Drive, La Jolla, CA 92093-0403, USA. 7 Department of Bioengineering, University of California, San Diego, 9500 Gilman Drive, La Jolla, CA 92093-0412, USA. ${ }^{凶}$ email: kzengler@ucsd.edu
} 
P hototrophic microbial communities exhibit symbiosis between photoautotrophic and heterotrophic organisms supported primarily by solar energy and the fixation of carbon dioxide $\left(\mathrm{CO}_{2}\right)$. This type of association dominates many biofilms, microbial mats, and lichens ${ }^{1-3}$, thriving in desiccation, nutrient starvation, and salinity or temperature extremes ${ }^{4}$. This ability to survive extreme conditions is due, in part, to division of labor and subsequent interactions between members of the community. Photoautotrophic members, classically either cyanobacteria or eukaryotic algae, convert $\mathrm{CO}_{2}$ into organic carbon for growth and maintenance of the heterotrophic partner(s). Exchange of these metabolites can sustain the heterotrophs under conditions devoid of any organic carbon source. In turn, the heterotrophs provide additional $\mathrm{CO}_{2}$, protection from environmental factors and predation, and often, a diverse array of metabolites produced by secondary metabolism ${ }^{5}$.

To date, understanding, engineering, and determining viable cultivation conditions for natural phototrophic communities remains challenging ${ }^{6}$. Thus, synthetic communities have been the primary platform for autotrophic-heterotrophic symbioses for bioenergy $^{7-11}$, resulting in novel phototrophic systems to produce biomass and value-added compounds, 12,13. Synthetic phototrophic communities (SPCs) have been utilized for the production of biofuels ${ }^{7}, a$-amylase, and polyhydroxyalkanoates among other compounds $8,10,12$. Complex communities consisting of algae and bacteria also have potential applications in waste-water treatment $^{14,15}$, bioremediation ${ }^{16}$, and as a bloom control method for phytoplankton ${ }^{17}$. Traditionally, microbial communities have been selected in long term adaptation and optimization experiments to define optimal culture conditions ${ }^{18}$. The critical challenge in synthetic community design is to maintain syntrophic interactions between members to avoid culture collapse ${ }^{14,19}$. Development of SPCs for bioproduction involves four critical steps: (a) strain selection, (b) screening of cultivation conditions, (c) efficient extraction of added-value products, and (d) process control and biomass recycling. The first three steps $(\mathrm{a}-\mathrm{c})$ are important drivers for implementation of successful bioproduction processes that can be optimized and guided using metabolic modeling.

Constraint-based metabolic modeling is a systems biology tool that provides a comprehensive metabolic understanding about individual microorganisms (metabolic models henceforth referred to as $\mathrm{M}$-models) ${ }^{20,21}$ and microbial communities (community-metabolic models henceforth referd to as CM-models) ${ }^{22-25}$. These models account for biochemical and genomic information for an individual or community at the genome-scale. Resulting models are solved using flux balance analysis and can accurately predict thousands of functional states ${ }^{24,26}$. Simulations performed with CM-models describe key metabolic functions of microbial communities, defining all possible interactions among partners based on genetic and/or metabolic fitness. CM-models also enable prediction of effective culture conditions for production in robust biotechnological processes ${ }^{22}$.

Here, we reconstruct CM-models encompassing bacteria and fungi that are important contributors to biofuel production. The cyanobacterium Synechococcus elongatus PCC7942, with its high growth rate and robust metabolism, is able to synthesize hydrogen, ethanol, 2,3-butanediol, sucrose, squalene, and fatty acids among other organic chemicals from light and $\mathrm{CO}_{2}{ }^{27}$. For heterotrophic partners, we focus on established microbial cell factories, i.e., Escherichia coli, Bacillus subtilis, and the fungus Yarrowia lipolytica. CM-models for four synthetic communities based on the sucrose-secreting $S$. elongatus in pairwise combination with E. coli (strains K-12 and W), B. subtilis str. 168, and $Y$. lipolytica Polg are deployed. Predictions about biomass and metabolites (methanol, formaldehyde, ethanol, butanal, and succinate) production yields as well as flux distributions through the metabolic networks are experimentally validated using physiological and gene expression data. We explore metabolic fitness and genetic stability by evaluating compensatory mechanisms in resistance to knockouts of genes with lethal phenotypic traits mimicking enzymatic damage. We test predictions experimentally for ten knockout strains and explore potential production capabilities of organic chemicals, such as methanol, formaldehyde, ethanol, butanal, and succinate for all microbial communities.

\section{Results}

Community-metabolic models predict members' growth rates. CM-models are a mathematical representation of genomic and metabolic knowledge of microorganisms within a community 22,23 . These models can be used to elucidate, understand, and generate hypotheses about biological mechanisms shaping interactions between community members ${ }^{22}$. CMmodels and M-models predict biological functions based on key substrate uptake capabilities referred to as constraints. Using the M-models of E. coli K-12 iML1515, E. coli W iJO1366W ${ }^{28,29}, B$. subtilis str. 168, iYO844 ${ }^{30}$, and Y. lipolytica Polg, iYali4 ${ }^{31}$ (Fig. 1a), we reconstructed four community-metabolic models by pairing each heterotroph with the model for S. elongatus iJB792 ${ }^{32}$. General properties of the community networks and M-models are shown in Table 1. The modeling compartment Shared Metabolite Pool (SMP) was manually curated in all CM-models, including experimental and genetic evidence of metabolic exchange capabilities by each community member. For example, we confirmed experimentally the consumption of 52 metabolites by $S$. elongates in monoculture. Experimental evidence, derived from highthroughput growth assays from consumption of amino acids, carbohydrates, and organic acids (BIOLOG Inc, Hayward, CA) was added into the SMP of all models. Metabolites in the SMP of all CM-models are listed in Supplementary Data 1 and simulation constraints for each CM-model are shown in Supplementary Data 2.

We simulated CM-models based on experimental constraints, such as the sucrose secretion rate of $0.182 \mathrm{mmol} \mathrm{gDW}^{-1} \mathrm{~h}^{-1}$ by an engineered $S$. elongatus strain ( $S$. elongatus $\left.\csc B^{+}\right)^{33}$. When this constraint was added into the M-model of S. elongatus (iJB792) the growth rate dropped about $16 \%$ from $0.0539 \mathrm{~h}^{-1}$ to $0.044 \mathrm{~h}^{-1}$, similar to experimental observations $\left(0.045 \pm 0.07 \mathrm{~h}^{-1}\right)$. Growth rate simulations and experimental results are shown in Fig. 1b. In monoculture, M-Models successfully predicted growth phenotypes of all five microbes in minimal media. Predictions of monocultures in minimal media with sucrose were similarly successful with the exception of Y. lipolytica. Since, Y. lipolytica cannot use sucrose as its sole carbon source we used an engineered strain ( $Y$. lipolytica $\mathrm{suc}^{+}$) with sucrose uptake capabilities in all the experiments shown here ${ }^{34}$.

Experimental validation of the CM-models yielded interesting and varied results. Predictions using the CM-models showed that S. elongatus $\left(\csc B^{+}\right.$or WT) should be able to grow at similar growth rates with $E$. coli or $B$. subtilis as in monoculture $(0.039 \pm$ $0.01 \mathrm{~h}^{-1}$ averaged across cocultures), but should grow faster when it is cultivated with $Y$. lipolytica $\left(0.062 \mathrm{~h}^{-1}\right)$. These expectations were validated by experiments with the exception of S. elongates$Y$. lipolytica communities in which S. elongatus did not see a boost in growth but instead exhibited diminished growth, despite our simulations showing active exchange of isoleucine and other amino acids.

Synthetic phototrophic communities (SPC) of S. elongatus $c s c B^{+}$ with each heterotrophic partner supported growth of both community members over $72-96 \mathrm{~h}$ (Fig. 1b). Surprisingly, this 
a

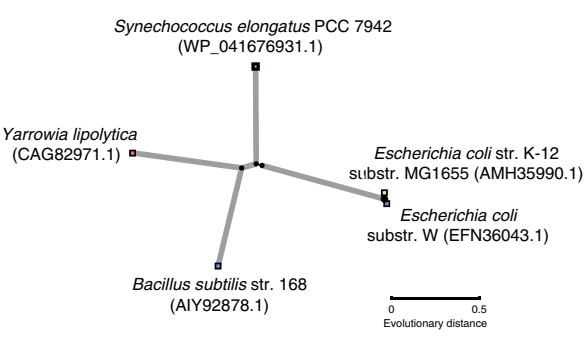

b

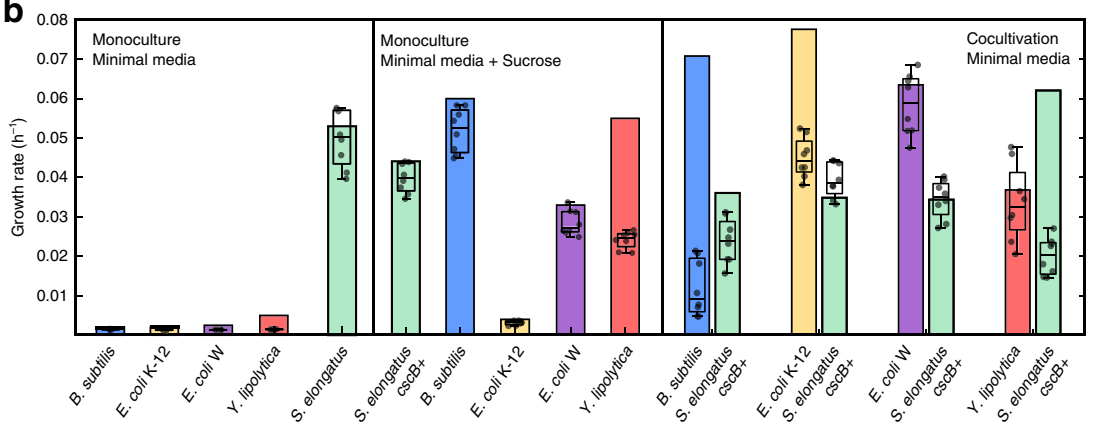

Fig. 1 Community members and growth performance. a Neighbor-joining tree based of almost full-length $16 \mathrm{~S}$ rRNA gene sequences, showing phylogenetic relationships between S. elongatus PCC7942 and the heterotrophic species used here. GenBank accession numbers are given in parentheses. b The bar plot depicts growth rates predicted using CM-models. Boxplots show experimental validation using at least six experimental replicates, the central mark indicates the median, and the bottom and top edges of the box indicate the 25th and 75th percentiles, respectively. The whiskers extend to the most extreme data points not considered outliers. The first panel shows growth in monoculture using minimal media without an organic carbon source. The second panel shows growth rates during monoculture, while using minimal media with additional sucrose. The third panel shows results obtained when combining S. elongatus $\csc ^{+}$with all heterotrophs in minimal media. Source data underlying Fig. $1 \mathrm{~b}$ are provided as a Source Data file.

Table 1 M-model and CM-model properties.

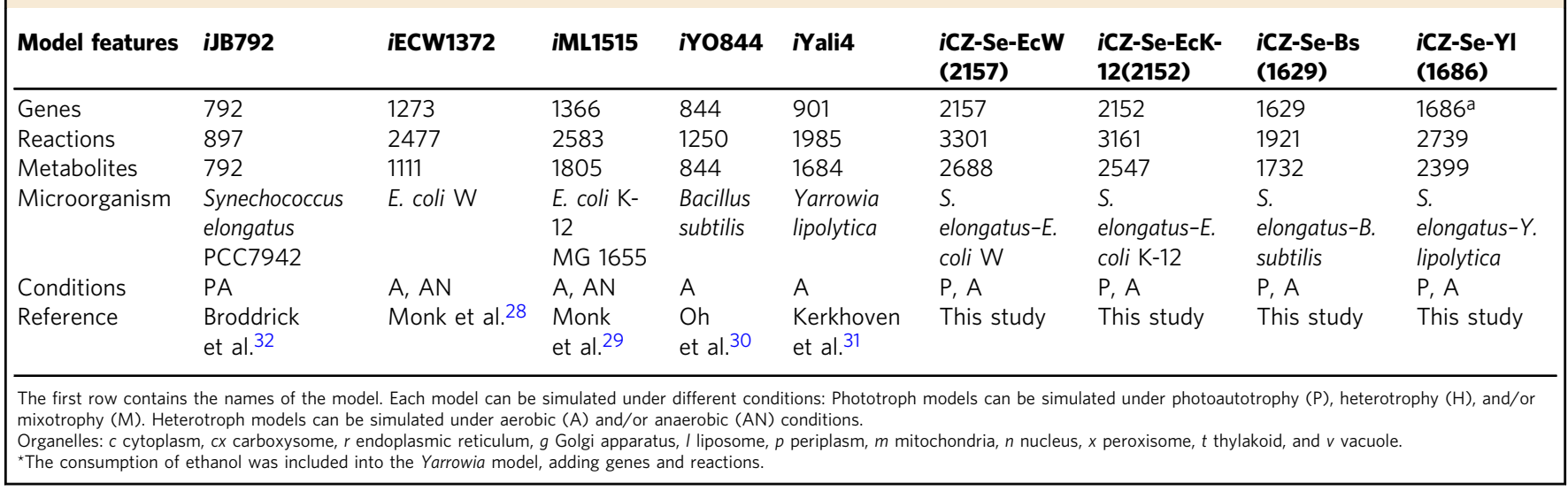

was even the case for $E$. coli $\mathrm{K}-12$, which is not able to consume sucrose. CM-model predictions of heterotrophic growth in communities with $S$. elongatus were most accurate for E. coli W and $Y$. lipolytica while E. coli $\mathrm{K}-12$ and B. subtilis experimental growth rates were slower than predicted. Experimental growth rates of phototrophs and heterotrophs ranged between $0.04 \pm 0.01$ $\mathrm{h}^{-1}$ and $0.06 \pm 0.015 \mathrm{~h}^{-1}$, similar to predicted growth rates of 0.05 $\mathrm{h}^{-1}$ and $0.07 \mathrm{~h}^{-1}$ (Fig. 1b). For the SPC containing B. subtilis we experimentally observed rapid growth of the heterotroph during the first $24 \mathrm{~h}$; however, this fast growth was not sustained over the course of growth $(72 \mathrm{~h})$. Growth curves for all SPCs are shown in Supplementary Fig. 1.

Predicted production capabilities underlie partner selection. CM-models offer a remarkably higher topological potential, achieving feasible solutions while optimizing for the synthesis of target metabolites by shifting the resources across members. CMmodels contain the most comprehensive collection of metabolites that are intermediates in the synthesis of biomass or are part of the biomass compositions (e.g., carbohydrates, lipids, and proteins) of the community members. Here, we simulated production capabilities of every metabolite in the models (9369 total, Fig. 2a). Metabolite production constraints were setup as boundaries synthesize each metabolite, thus community growth simulations were calculated by maximizing the biomass growth of both community members at the same time. We considered metabolite production feasible when metabolite boundaries enabled the growth of both community members (Fig. 2a). We found community-specific production potential in all SPCs; the SPC containing E. coli K-12 was capable of producing the most metabolites (111 metabolites), followed by E. coli W (106 metabolites), Y. lipolytica (60 metabolites), and B. subtilis (39 metabolites).

We evaluated the capability of community members to secrete organic chemicals (methanol, formaldehyde, ethanol, butanal, and succinate), that are declared as primary feedstock for chemical synthesis by the Environmental Protection Agency of the United States of America. Currently these metabolites are manufactured primarily through either chemical synthesis or single-strain biorefineries ${ }^{35,36}$. Utilization of these metabolites accounts for $4-30 \%$ of the total production costs of feedstocks ${ }^{35}$. When we evaluated the maximum production capability, growth phenotypes, metabolic exchange, and yields attained with the CM-Models during the production of organic chemicals (Fig. 2b and Supplementary Fig. 2), we predicted differences in the metabolic capabilities of each SPC. For example, we found that all SPCs were theoretically able to synthesize ethanol $(0.011 \pm 0.01 \mathrm{~g}$ $\left.\mathrm{gDW}^{-1}\right)$, or succinate $\left(0.066 \pm 0.004 \mathrm{~g} \mathrm{gDW}^{-1}\right)$, but only SPCs containing $E$. coli strains were able to synthesize formaldehyde (average yield $0.017 \mathrm{~g} \mathrm{gDW}^{-1}$ ) and butanal (average yield 0.012 
a

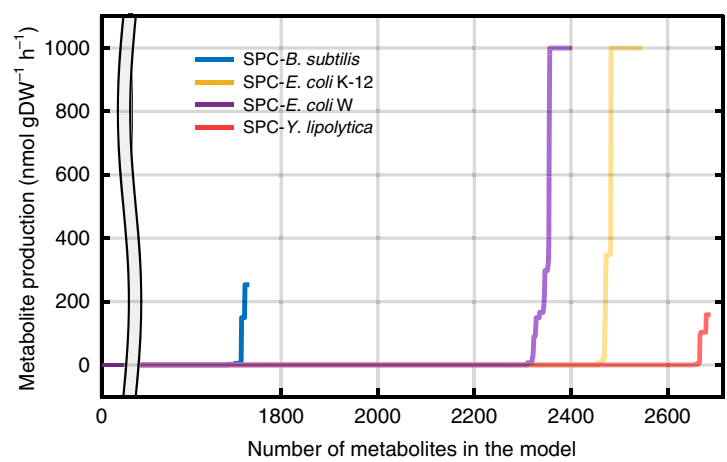

C

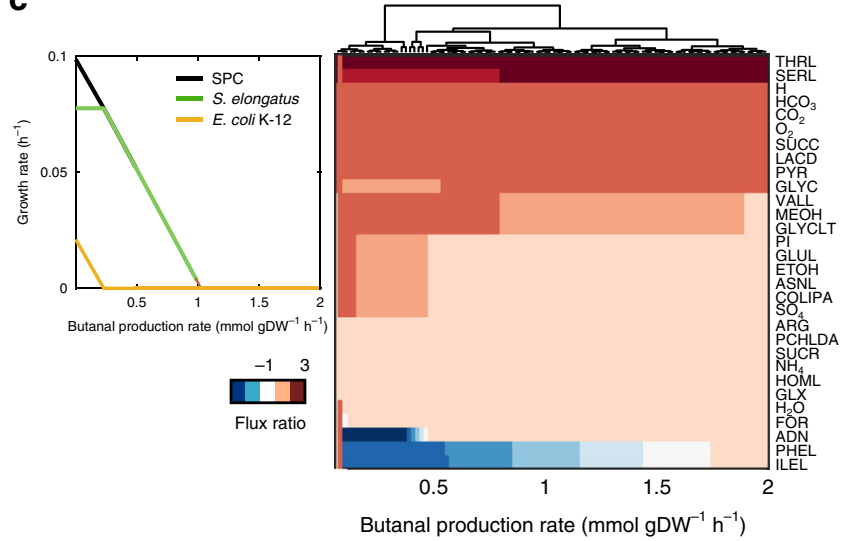

b

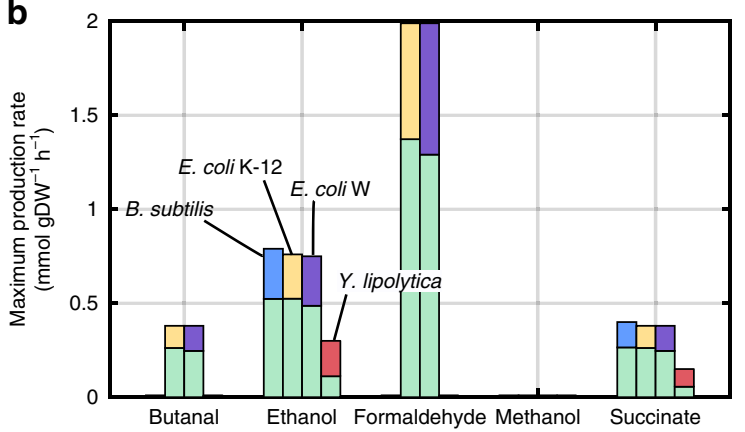

d

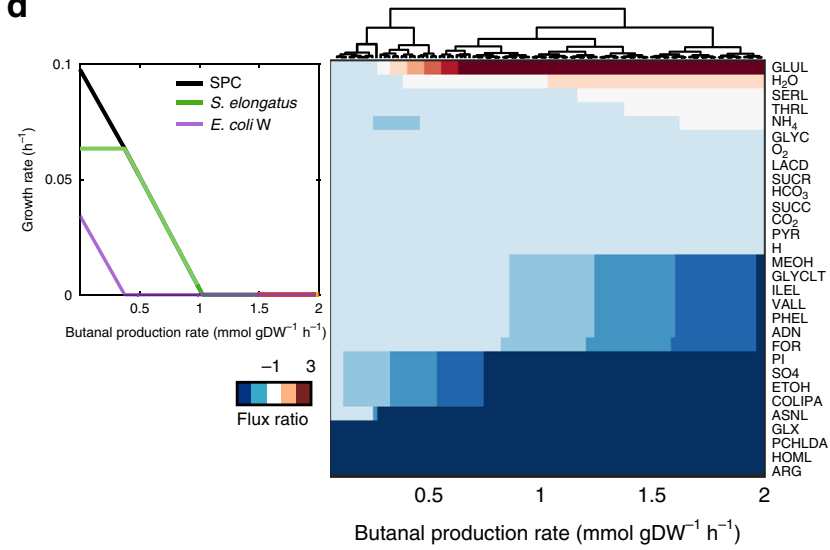

Fig. 2 Organic chemical production by synthetic phototrophic communities. a Production capabilities were evaluated for all SPCs by iteratively simulating the synthesis of metabolites while maximizing for growth. Metabolite production rates were sorted and plotted for each synthetic phototrophic community (SPC). Metabolites feasible to produce were identified by having production rates over $1 \times 10^{-2} \mathrm{mmol}_{\mathrm{gDW}}-1 \mathrm{~h}^{-1}$. b Overall production capabilities for butanal, ethanol, formaldehyde, methanol, and succinate. The bar plot shows the predicted maximum production rate achieved by each SPC. The predicted abundance (growth rate) of community members is shown for S. elongatus $\csc B^{+}$(green) and for heterotrophs (blue B. subtilis, yellow $E$. coli $\mathrm{K}-12$, purple $E$. coli W, or pink Y. lipolytica). Microbial communities containing $E$. coli strains showed high formaldehyde production potential. c, d Predicted growth rates and associated metabolic exchanges for the SPC composed of S. elongatus $\csc B^{+}$and either E. coli K-12 (c) or E. coli W (d) while producing butanal. CMmodels enable prediction of substrate resource allocation into growth or into target metabolites. Production of metabolites reduces the growth of community members as observed in the growth rate plots. Here, the overall growth rate of the community (black line) and each member (green $S$. elongatus $\csc B^{+}$paired with yellow or purple for $E$. coli $\mathrm{K}-12$ or $E$. coli $\mathrm{W}$, respectively) were simulated. Simulations show that at higher butanal production rates the heterotroph will be outcompeted by the phototroph before the SPC crashes. The complete-linkage clustering based on the metabolic exchange predictions shows associations among metabolites listed on the $y$-axis and butanal production rates from left to right. Growth rate predictions were remarkably associated with a specific metabolic exchange. For example, the synthesis of butanal by the E. coli K-12 SPC was possible by increasing the flux exchange of metabolites such as isoleucine (ILEL), phenylalanine (PHEL), serine (SERL), and threonine (THRL), while for the E. coli W SPC butanal production relied heavily on L-glutamine exchange (GLUL). Metabolic exchanges of all SPCs are given in Supplementary Fig. 3.

$\pm 0.002 \mathrm{~g} \mathrm{gDW}^{-1}$ ) (Fig. 2c, d). Metabolite productivities were compared with engineered strains under monoculture and wildtype coculture conditions, showing that cocultures are more competitive to produce succinate but not ethanol (Supplementary Data 3). Thus, using engineered strains in cocultivation could potentially achieve even higher productivities.

We found that community growth rate is driven by memberspecific flux distributions and metabolic exchange, which responds differently to the requirement to produce an additional organic compound. For example, differences in the predicted flux through the SMP at each production rate of butanal were observed (Fig. 2c, d). In the community containing E. coli $\mathrm{K}-12$, the model predicted that $S$. elongatus $c s c B^{+}$would increase the flux exchange of serine and threonine over two-fold, resulting in higher secretion of these metabolites. In turn, E. coli K-12 would provide adenine, phenylalanine, and isoleucine at higher fluxes (Fig. 2d). Metabolic adjustments also hinted at new metabolites being exchanged (e.g., ammonium, arginine, succinate, and glyoxylate) during butanal secretion. The exchange of metabolites was activated by butanal secretion since these metabolites were not exchanged when maximizing only for biomass production. Simulations regarding the SPC with E. coli W showed an overall reduction of the exchange flux of all transport reactions except for glutamine. This effect was associated with depletion of the heterotrophic partner (Fig. 2d). Although E. coli K-12 and E. coli $\mathrm{W}$ are genetically very similar, their individual metabolic networks harbor unique metabolic capabilities. E. coli K-12 has 163 unique reactions and $E$. coli $\mathrm{W}$ has 180 . While the main differences were found in alternative carbon metabolism, the metabolic networks also differed in the number of reactions in cysteine, glutamate, tyrosine, and valine metabolism, lipopolysaccharide biosynthesis, and in active transporters (Supplementary Data 4).

Community members interact through adjusting their fluxes associated with metabolic exchange and/or with specific metabolic pathways. In addition to $\mathrm{CO}_{2}$ and $\mathrm{O}_{2}$, the models predicted a wide array of other metabolites, including alcohols, organic acids, and amino acids, would be exchanged in order to maximize growth. Overall, the CM-models identified 16 exchanged metabolites in all SPCs (Fig. 3a, Venn diagram). These included 
a

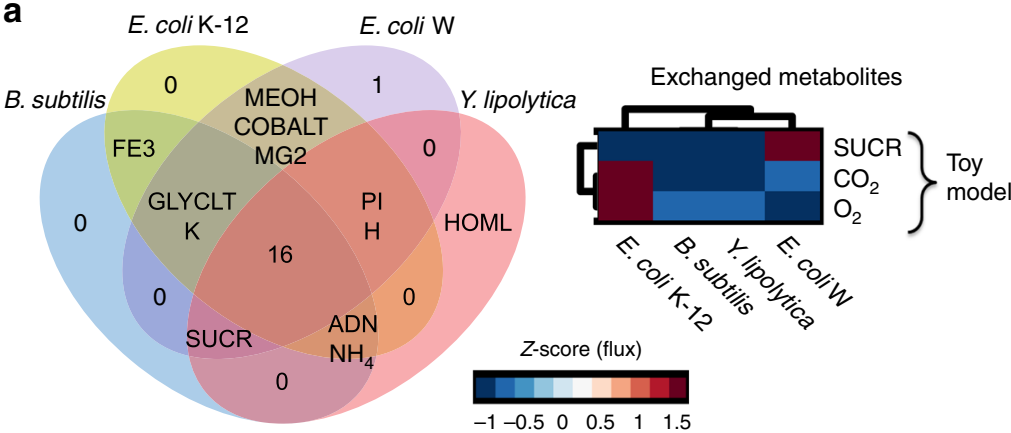

Flux predictions by SCP and experimentally measured metabolites (*)
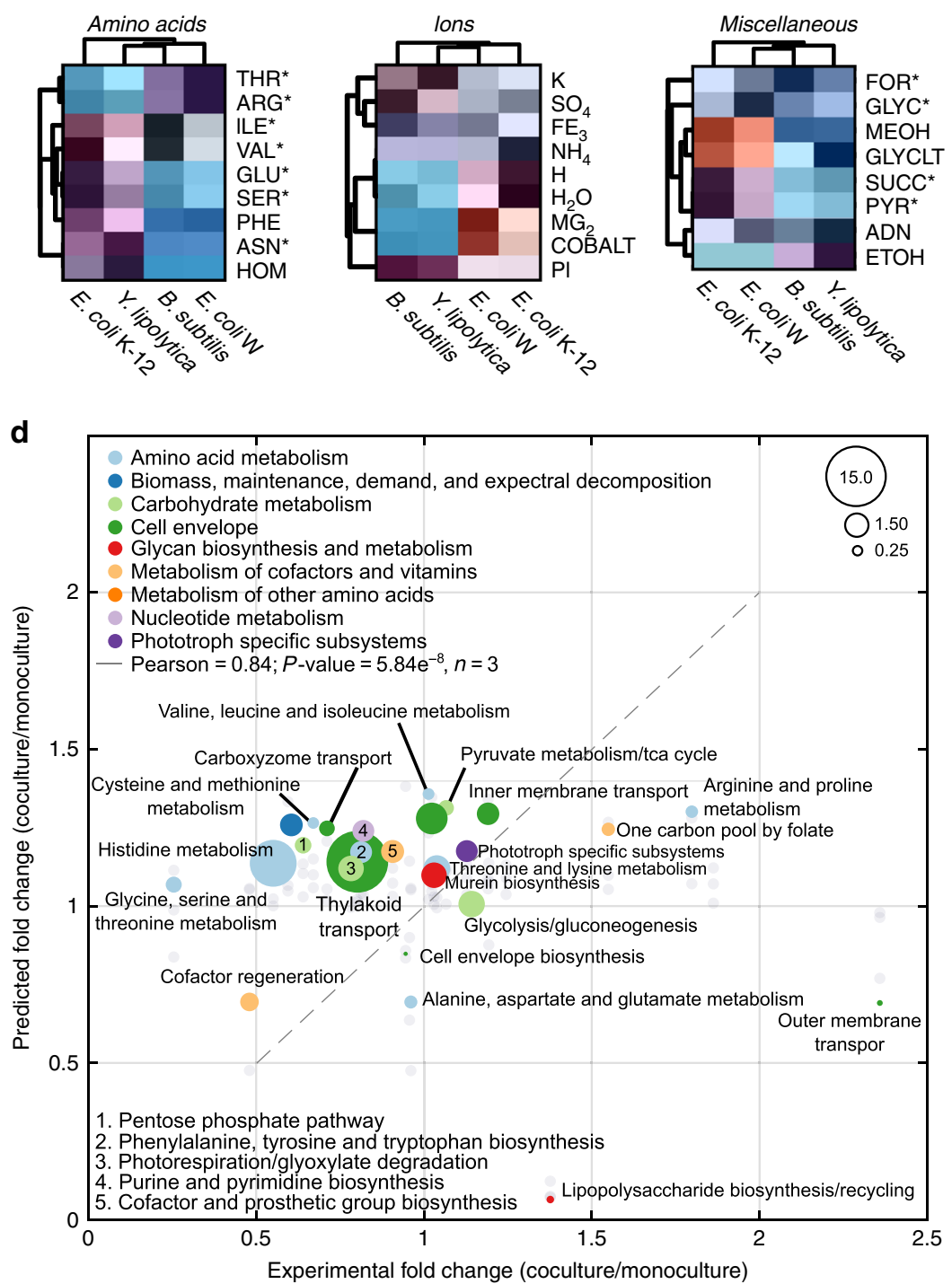

b

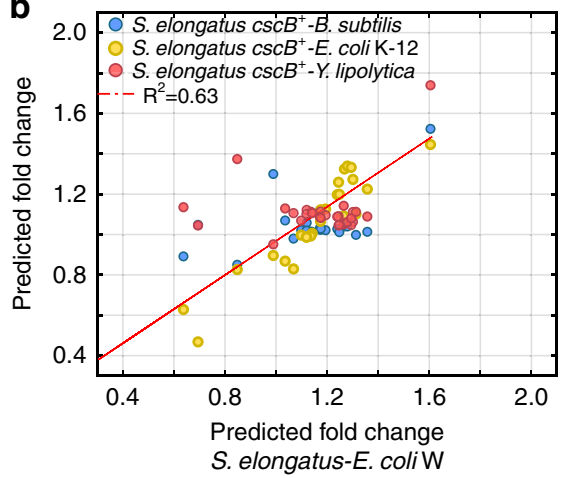

c

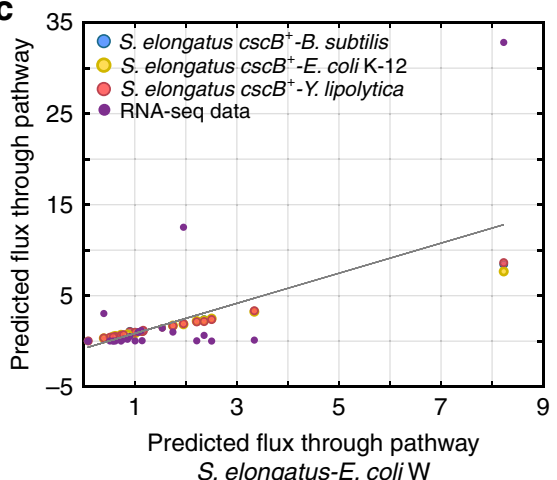

metabolites supplemented in the culture medium as well as biologically produced metabolites, such as threonine, isoleucine, and pyruvate (highlighted in bold in Fig. 3). Additionally, most of the SPCs exchanged metabolites specific for a particular SPC, such as homoserine for the community composed of $S$. elongatus and Y. lipolytica, and arginine for S. elongatus and E. coli W. Predicted fluxes associated with metabolic exchanges were clustered into three groups, i.e., ions, amino acids, and miscellaneous (organic acids, overflow metabolism and carbohydrates). We predicted that the SPC containing E. coli $\mathrm{K}-12$ was the most efficient in exchanging amino acids based on its total metabolite exchange flux, e.g., isoleucine, valine, glutamate, serine, and phenylalanine (Fig. 3a, clustergram). Similar predictions about higher fluxes were obtained for ions and miscellaneous metabolites. The SPC with the second highest exchange fluxes was $S$. elongatus $\operatorname{cscB}+$ and $E$. coli W, which was characterized by high exchanges of threonine, arginine, and isoleucine. This SPC was the most efficient in activating exchange reactions containing magnesium and cobalt. The total fluxes in the SPCs containing B. subtilis or Y. lipolytica were lower in comparison to fluxes of SPCs containing E. coli. The B. subtilis and Y. lipolytica containing SPCs clustered together and exhibit 
Fig. 3 Metabolic exchange is linked to differential expression in RNA-seq data and predicted flux distributions. a The Venn diagram shows the predicted metabolic exchange for all synthetic phototrophic communities. Metabolites highlighted in bold represent the 16 (e.g., ASNL, GLUL, and ILEL) metabolites shared by all synthetic communities. Besides expected metabolites to be exchanged $\left(\mathrm{CO}_{2}, \mathrm{O}_{2}\right.$, and sucrose), simulations show mutual transfer of amino acids, ions, and other metabolites. Marked metabolites $\left(^{\star}\right)$ were identified in supernatant samples using target metabolomics (Tables 2, 3).

b $S$. elongatus $\csc B^{+}$fold-change analysis. Correlation between predicted subsystems fold-change of $S$. elongatus under cocultivation with $E$. coli W ( $x$-axis) versus predicted fold-change when cultivated with $B$. subtilis (Pearson $=0.49, p$-value $=0.011, n=3$, blue dots), $E$. coli $\mathrm{K}-12$ (Pearson $=0.93, p$-value $=$ 9.1e-12, $n=3$, yellow dots), and Y. lipolytica (Pearson $=0.33, p$-value $=0.009, n=3$, red dots). c Predicted flux for each pathway in $S$. elongatus cocultured with $E$. coli W (x-axis) and other heterotrophic partners ( $y$-axis) including B. subtilis, E. coli W, and Y. lipolytica. Flux evaluated by RNA-seq of S. elongatus cocultured with $E$. coli W (purple) demonstrates validation of flux prediction (Pearson $=0.82, p$-value $=1.02 \mathrm{e}^{-7}, n=3$ ). $\mathbf{d}$ Fold-change of RNA-seq data and predicted flux distributions under mono- and coculture was calculated and normalized at the pathway level. Expression data were obtained for the SPC containing E. coli W. Bubble size represents the median of expression burden and flux burden by pathway. Predicted fold-change for the microbial communities containing B. subtilis, E. coli W, and Y. lipolytica is shown in light gray dots.

remarkably different flux profiles compared to E. coli K-12 (see also Supplementary Figs. 3, 4).

Interestingly, the SPC composed of S. elongatus $\mathrm{cscB}^{+}$and $E$. coli $\mathrm{K}-12$, which is unable to grow with sucrose, was predicted to thrive in minimal media devoid of any organic carbon source. These predictions were validated experimentally and we identified E. coli $\mathrm{K}-12$ as the most suitable heterotrophic partner for $S$. elongatus, exhibiting the highest growth phenotypes. We therefore characterized the metabolic and genetic interactions of $S$. elongatus $\operatorname{cscB}^{+}$and $E$. coli $\mathrm{K}-12$ in greater detail.

Metabolic profiles give insight into microbial interactions. Metabolic interactions were experimentally characterized using Nuclear Magnetic Resonance (NMR) and Gas ChromatographyMass Spectrometry (GC-MS). Metabolic profiles for 34 metabolites were measured from biomass samples and culture medium supernatants under mono- and coculture conditions. Experimentally determined metabolic profiles validated the exchange of predicted metabolites. NMR and GC-MS analysis of intracellular (Table 2) and extracellular (Supplementary Data 5) metabolites for the SPC of S. elongatus $\operatorname{cscB} B^{+}$and E. coli K-12 along with monoculture profiles was performed. Sucrose remained constant in the extracellular medium of $S$. elongatus $\csc B^{+}$monoculture and the $S$. elongatus $\csc B^{+}-E$. coli $\mathrm{K}-12$ coculture due to the lack of a sucrose transporter in this $E$. coli strain. Other metabolites detected in the extracellular broth of this SPC (S. elongatus $\csc B^{+}$. E. coli $\mathrm{K}-12$ ) were pyruvate, methanol, formate, acetate, and high concentrations $(13.7 \mathrm{mM})$ of ethanol, suggesting that these are also available for metabolite exchange. These metabolites were often lower in coculture than in monocultures, providing further support of this exchange.

Our computational studies suggested optimized growth should include the exchange of a number of additional metabolites, especially amino acids (Fig. 3a). In order to determine if these amino acids and other metabolites were present in cells, we evaluated intracellular metabolites in S. elongatus $\csc B^{+}$and $E$. coli K-12 using GC-MS. Some intracellular metabolites, including the amino acids glutamate and aspartate and the dipeptide glycylproline (precursor of serine and threonine synthesis) accumulated to higher relative levels in S. elongatus compared to $E$. coli. Other metabolites, such as malonate, were detected intracellularly as well. GC-MS analysis (Table 3) confirmed the high intracellular levels of glutamate and aspartate as well as other amino acids in S. elongatus, and also revealed high intracellular levels of glycerol and glycine in S. elongatus relative to its $E$. coli $\mathrm{K}-12$ partner. Interestingly all these metabolites were confirmed experimentally to be effectively transported into the cytoplasmic space of S. elongatus and E. coli K-12 (Supplementary Data 1). Overall, our NMR and GC-MS analysis revealed a different intracellular metabolic fingerprint for the cyanobacterium as compared to its heterotrophic partner. This provides further evidence that phototrophic communities can be advantaged through division of labor using interwoven metabolic networks, especially in alleviation of partner-specific bottlenecks.

Simulated pathway fluxes suggest network bottlenecks. CMModels not only unravel metabolic exchange but can also decipher alterations in the activity of complete pathways. Shifting from mono- to coculture conditions triggered changes across fluxes in multiple pathways of the entire network. Our predictions suggested that these metabolic changes occur in all SPCs studied here. The predicted fold-change (coculture/monoculture) of subsystem fluxes in all SPCs were remarkably correlated (Pearson correlation, average $R^{2}=0.63$ ) (Fig. $3 \mathrm{~b}, \mathrm{c}$ ). Similarly, the predicted flux through pathways were well correlated across all SPCs and significantly correlated with S.elongatus and E.coli W expression data (Pearson $=0.82, p$-value $=1.02 \mathrm{e}^{-7}$ ).

Predicted flux differences between mono- and coculture conditions were identified in transport reactions and metabolism of pyruvate, purine, pyrimidine, alanine, aspartate, and glutamate in both members of the SPC (Fig. 3d). Furthermore, flux through member-specific pathways, such as photosynthesis, carbon fixation, and biosynthesis of plastoquinol, phylloquinone, and carotenoids, significantly changed between mono- and coculture conditions. Measured expression data of the SPC containing $S$. elongatus and $E$. coli $\mathrm{W}$ showed significantly differential expression (two sided $t$-test, $n=3, p$-value $<0.05$ ) of genes associated with alanine, aspartate, and glutamate metabolism, arginine and proline metabolism, the pentose phosphate pathway, phototroph-specific metabolism, purine and pyrimidine metabolism, and transport reactions as predicted (Supplementary Fig. 5 and Supplementary Data 7).

We quantified and compared expression and fluxes by pathway, identifying potential bottlenecks that if relieved could improve biomass yields. For example, predicted flux and observed expression data associated with transporters located in the thylakoid as well as histidine metabolism in the cytoplasm were highly activated, making up around $50 \%$ of the total expression (RNA-seq reads) and flux (mmol gDW ${ }^{-1} \mathrm{~h}^{-1}$ ) across the entire metabolic network. Histidine metabolism is linked with the pentose phosphate pathway and alanine, aspartate, and glutamate metabolism, encompassing the synthesis of several glutamate precursors. Even though we could not quantify/detect intracellular accumulation of histidine experimentally, glutamine, a histidine precursor, was accumulated in both $S$. elongatus $(9.9 \mathrm{mM})$ and E. coli $\mathrm{K}-12(2.1 \mathrm{mM})$. This metabolite was not found in the supernatant of any mono- or cocultures. Overall, CM-models are a reliable tool to identify functional states in association with growth phenotypes, offering details of cellular communication and potential network bottlenecks limiting microbial interactions. 
Table 2 NMR data for extracellular metabolite concentrations.

\begin{tabular}{|c|c|c|c|c|}
\hline Metabolite & Metabolite ID & E. coli K-12 ( $\mu \mathrm{M})$ & Coculture $(\mu \mathrm{M})$ & S. elongatus ( $\mu \mathrm{M})$ \\
\hline 2-oxoglutarate & akg & 404.200 & 0.000 & 0.000 \\
\hline 3-phosphoglyceric acid & g3pg & NF & NF & NF \\
\hline 4-hydroxybenzoate & $4 \mathrm{hbz}$ & 12.800 & 0.000 & 0.000 \\
\hline Acetate & $\mathrm{ac}$ & 424.000 & 2.300 & 3.400 \\
\hline Acetone & aact & 7.300 & 4.400 & 7.200 \\
\hline Adenine & ade & 0.000 & 0.000 & 0.000 \\
\hline Citric acid & cit & NF & NF & NF \\
\hline D-arabinose & arab & NF & NF & NF \\
\hline D-fructose & fru & NF & NF & NF \\
\hline D-fructose-6-phosphate & $f 6 p$ & NF & NF & NF \\
\hline D-glucose & glc & 0.000 & 0.000 & 0.000 \\
\hline D-glucose-6-phosphate & g6p & NF & NF & NF \\
\hline D-mannitol & $\mathrm{mnl}$ & NF & NF & NF \\
\hline D-ribose & rib & NF & NF & NF \\
\hline D-ribose-5-phosphate & r5p & NF & NF & $\mathrm{NF}$ \\
\hline Ethanol & etoh & 13.200 & 13.700 & 18.600 \\
\hline Formate & for & 1343.200 & 6.500 & 43.300 \\
\hline Glycerol & glyc & NF & NF & NF \\
\hline Glycine & gly & NF & NF & NF \\
\hline Isobutyrate & but & 2.300 & 0.000 & 0.000 \\
\hline L-aspartic acid & asp & 0.000 & 0.000 & 0.000 \\
\hline L-glutamic acid & glu & 0.000 & 0.000 & 0.000 \\
\hline L-serine & ser & NF & NF & NF \\
\hline L-threonine & thr & 0.000 & 0.000 & 0.000 \\
\hline L-valine & val & 0.000 & 0.000 & 0.000 \\
\hline Methanol & meoh & 1.600 & 6.700 & 5.400 \\
\hline Pantothenate & pnto & 5.800 & 0.000 & 0.000 \\
\hline Proline & pro & NF & NF & NF \\
\hline Propylene glycol & glyclt & 4.700 & 0.000 & 0.000 \\
\hline Pyruvate & pyr & 0.000 & 0.000 & 2.600 \\
\hline Succinate & succ & 5.400 & 0.000 & 0.000 \\
\hline Sucrose & sucr & 903.500 & 86.000 & 101.500 \\
\hline Tyrosine & tyr & 0.000 & 0.000 & 0.000 \\
\hline Lactate & lac & 0.000 & 0.000 & 0.000 \\
\hline
\end{tabular}

SPCs are more resistant to lethal traits than monocultures. Tolerance to inhibitors is one of the main challenges for strain engineering of monocultures. Often growth rates and productivities are limited by the accumulation of toxic intermediates (e.g., end-products and/or metabolic intermediates) that can harm productivity or lead to culture collapse ${ }^{37}$. These inhibitions can affect metabolism at the reaction or pathway levels ${ }^{38}$. To probe the ability of SPCs to cope with enzymatic inhibition, we simulated single gene deletions of all genes in the CM-models (7624 total).

Model simulations showed that members of microbial communities can efficiently compensate for some metabolic activities, overcoming up to $27 \%$ of phenotypic traits lethal to one member, allowing the damaged participant to thrive in the community though they could not in monoculture. For example, during monoculture growth of $S$. elongatus $c s c B^{+}, 350$ genes are essential, meaning if they are knocked out $S$. elongatus $\csc B^{+}$cannot grow in minimal media. This is in contrast to $S$. elongatus $\csc B+$ grown in communities where the number of lethal deletions falls to 327 or 326 , depending on the cocultivation partner (Fig. $4 \mathrm{a}$ ). This equates to a $7 \%$ increase in community resilience in the face of gene knockouts as a proxy for inhibited genes. So despite $S$. elongatus $\csc B^{+}$being the growth-sustaining microorganism in the community, our predictions showed that the presence of $E$. coli strains could compensate for up to $7 \%$ of lethal knockouts in S. elongatus $\csc B^{+}$.
We used these genome-scale model simulations to guide the selection of ten $E$. coli $\mathrm{K}-12$ knockout $(\mathrm{KO})$ mutants with variable growth phenotypes in monoculture versus coculture conditions to test experimentally. Six of those mutants were predicted to not grow in monoculture and were associated with amino acid metabolism (ilv $\mathrm{C}$, ilv $\mathrm{E}$, and phe $\mathrm{A})$, cofactor and prosthetic group biosynthesis (cysG and thrC), and inner membrane transport (phoE). Of these, most strains died in monoculture in minimal media as expected and only the strain lacking ilvE grew in monoculture under our cultivation conditions (Fig. 4b). Four strains with non-essential genes knocked out were associated with amino acid metabolism (aspC), oxidative phosphorylation (atpC), the pentose phosphate pathway (talB), and inner membrane transport (cycA) (Fig. 4b, Supplementary Data 8,9). All of these strains grew successfully in monoculture albeit with grow rates that varied significantly with respect to the predicted rates, since we used uptake rates determined under wild-type conditions to constrain the CM-model.

Growth of E. coli strains missing ilvC, cys G, pheA, and thrC was rescued by $S$. elongatus $c s c B^{+}$as predicted for the strains ilvC and pheA (Supplementary Data 9). The genetic damage in the heterotrophic partner did not significantly affect the growth rate of the phototroph experimentally (Fig. $4 \mathrm{~b}$, two sided $t$-test, $n=4$, $p$-value $>0.05)$. The model demonstrated good accuracy for growth/no-growth prediction of the ten assayed KOs. Matthews correlation coefficient (MCC) values were estimated for each 


\section{Table 3 Intracellular metabolite Z-score comparisons from} GC-MS.

\begin{tabular}{|c|c|c|c|}
\hline Metabolite & Metabolite ID & $\begin{array}{l}\text { E. coli K- } \\
12(Z- \\
\text { score) }\end{array}$ & $\begin{array}{l}\text { S. elongatus } \\
\text { (Z-score) }\end{array}$ \\
\hline 2-oxoglutarate & akg & 2.077 & 0.085 \\
\hline 3-phosphoglyceric acid & g3pg & -0.391 & 2.258 \\
\hline 4-hydroxybenzoate & $4 \mathrm{hbz}$ & -0.535 & -0.418 \\
\hline Acetate & $\mathrm{ac}$ & $\mathrm{NF}$ & NF \\
\hline Acetone ${ }^{a}$ & aact & 1.943 & -0.917 \\
\hline Adenine & ade & 1.575 & 1.072 \\
\hline Citric acid & cit & -0.428 & -0.963 \\
\hline D-arabinose & arab & 1.759 & -0.591 \\
\hline D-fructose & fru & 0.953 & -0.546 \\
\hline D-fructose-6-phosphate & $f 6 p$ & 2.069 & 0.571 \\
\hline D-glucose & glc & 2.212 & -0.343 \\
\hline D-glucose-6-phosphate & g6p & 1.618 & 1.297 \\
\hline D-mannitol & $\mathrm{mnl}$ & 2.268 & -0.378 \\
\hline D-ribose & rib & 2.262 & -0.363 \\
\hline D-ribose-5-phosphate & r5p & -0.220 & 2.262 \\
\hline Ethanol & etoh & NF & NF \\
\hline Formate & for & NF & NF \\
\hline Glycerol & glyc & -0.372 & 1.599 \\
\hline Glycine & gly & 0.178 & 2.199 \\
\hline Isobutyrate & but & 2.268 & -0.378 \\
\hline L-aspartic acid & asp & 0.277 & 2.173 \\
\hline L-glutamic acid & glu & 0.210 & 2.192 \\
\hline L-serine & ser & 1.390 & -0.585 \\
\hline L-threonine & thr & -0.370 & 2.264 \\
\hline L-valine & val & NF & NF \\
\hline Methanol & meoh & NF & $\mathrm{NF}$ \\
\hline Pantothenate & pnto & 0.951 & -0.410 \\
\hline Proline & pro & -0.378 & 2.268 \\
\hline Glycolate $^{b}$ & glyclt & 0.135 & 0.784 \\
\hline Pyruvate & pyr & 2.249 & -0.142 \\
\hline Succinate & succ & 2.263 & -0.429 \\
\hline Sucrose & sucr & -0.179 & -0.546 \\
\hline Tyrosine & tyr & 0.247 & 1.936 \\
\hline Lactate & $\mathrm{lac}$ & 2.248 & -0.196 \\
\hline \multicolumn{4}{|c|}{$\begin{array}{l}\text { Z-score was determined using three independent biological replicates. Standard error of all } \\
\text { measurements is below } 10 \% \text { of the average observed concentration. } \\
\text { NF not found. } \\
\text { Metabolite precursor found: } \\
\text { aAcetoacetate. } \\
\text { bPropylene glycol. }\end{array}$} \\
\hline
\end{tabular}

condition, by comparing experimental and predicted outcomes (Supplementary Data 9). Across KOs, the highest prediction accuracy was observed for E. coli K-12 monoculture (0.90), followed by S. elongatus $c s c B^{+}$coculture (0.7) and E. coli K-12 coculture (0.7); the positive predicted values ranged from 0.83 to 1 for all conditions. Selected strains were also cultured in rich medium ( $80 \%$ Luria-Bertani (LB) $+20 \%$ BG-11). We observed that all KOs were rescued in monoculture. Interestingly, the phototrophic communities maintained syntrophic interactions despite the capability of $E$. coli to thrive in rich medium. Overall, the growth rates of community and monoculture increased up to $41 \%$ while cultured in rich medium (see Supplementary Fig. 6).

Predicted metabolic exchange was highly reciprocal among community members, meaning that the secretion flux was identical to the uptake flux by the partner for most metabolites under optimal conditions. However, simulations also showed additional secretion of metabolites by $E$. coli $\mathrm{K}-12$, such as lactate and citrate for all KO strains. Resistance to lethal phenotypic traits is associated with metabolite-tuning and metabolite-fluxactivation under specific conditions. For example, simulations of the E. coli K-12 pheA gene $\mathrm{KO}$ promoted increased uptake of $\mathrm{O}_{2}$ from S. elongatus, while E. coli K-12 increased uptake of threonine, succinate, valine, glycerol, adenosine, phenylalanine, and asparagine while secreting $\mathrm{CO}_{2}$, glycolate, pyruvate, glucose, serine, formate, isoleucine, and deactivating the exchange of leucine and ethanol (Fig. 4c).

This advanced community modeling tool gives insight into stability of these cultivation platforms, namely through enhanced tolerance to inhibition through offsetting lethal traits among community members. Future network discoveries of individual microorganisms will continue improving the definition of constraints as well as the predictive power of M-models and eventually of CM-models. Such discoveries will highlight areas of interest that may be responsible for current mismatches between predictions and experimental results.

\section{Discussion}

Emergent metabolic modeling tools have enabled significant progress toward understanding interactions in microbial communities. This achievement, together with advances in various omics methodologies, has opened the possibility of switching from biorefineries consisting of monocultures to multiorganismal cell factories 22,25 . Currently, a number of challenges to multi-organismal cell factories remain, such as high sensitivity to cultivation collapse under unexpected conditions and/or for unknown reasons $7,14,26$. Utilization of sustainable microbial communities (e.g., SPCs) has resulted in a promising approach to overcome current limitations ${ }^{7,10,11}$. Furthermore, the use of both synthetic and natural phototrophic communities allows for the utilization of renewable resources for sustainable production ${ }^{39}$, maintaining diverse and robust microbial interactions, reducing overall cost of feedstocks and minimizing transport logistics ${ }^{35}$. We explored the potential of photosynthesis-based processes as sustainable growth. Simulations predicted different growth phenotypes depending on the heterotrophic partner (B. subtilis, E. coli K-12, E. coli W, or Y. lipolytica). The SPCs containing E. coli improved community growth effectively by exchanging metabolites (e.g., asparagine, glutamine, phenylalanine, pyruvate, serine, succinate, and valine, among others, Figs. 2, 3) to take maximum advantage of supplied nutrients and light for the production of organics, as predicted (Fig. 2b, d). Meanwhile, SPCs containing $B$. subtilis or Y. lipolytica did not grow experimentally as predicted, possibly due to the high sensitivity of amino acids to folding events of metal transporters ${ }^{40}$, deactivation of hexose transporters in Y. lipolytica ${ }^{41}$, as well as to the presence of reactive oxygen species ${ }^{7,8}$ potentially affecting growth of $B$. subtilis. Furthermore, sugar assimilation in $Y$. lipolytica, including the uptake mechanism, metabolic pathways, and regulatory mechanisms, is currently poorly understood ${ }^{26,41}$.

Predicted member fitness depends on specific key functions that maintain and enhance the community performance of the SPC, especially in pathways regarding arginine and proline metabolism, citric acid cycle, one-carbon and folate metabolism, and pyruvate metabolism (Fig. 3b). Accurate prediction of metabolic adjustment and fitness improvement in coculture conditions offers a portrait of integral usage of light, $\mathrm{CO}_{2}$, and $\mathrm{O}_{2}$ in addition to other metabolites, such as alcohols, organic acids, and amino acids that are naturally produced during cultivation and can enhance community performance. Synthesis of metabolites associated with overflow and secondary metabolism (e.g., formate, ethanol, and acetate, Supplementary Figs. 3, 4) is prevented as much as possible in monocultures ${ }^{42}$ or controlled by the energetic state of cells ${ }^{43}$. However, for microbial communities, the active exchange of these overflow metabolites maintains and promotes the growth of community members. Certain metabolites predicted to be part of the metabolic exchange in this study 
a
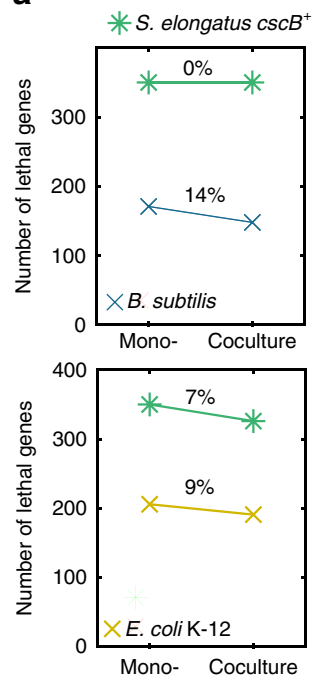

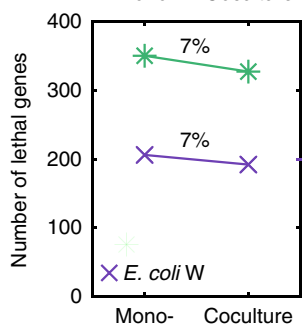

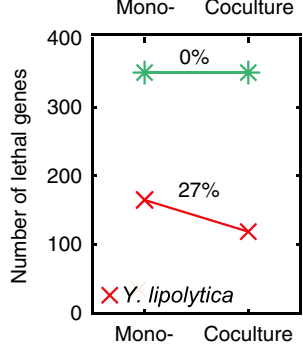

b

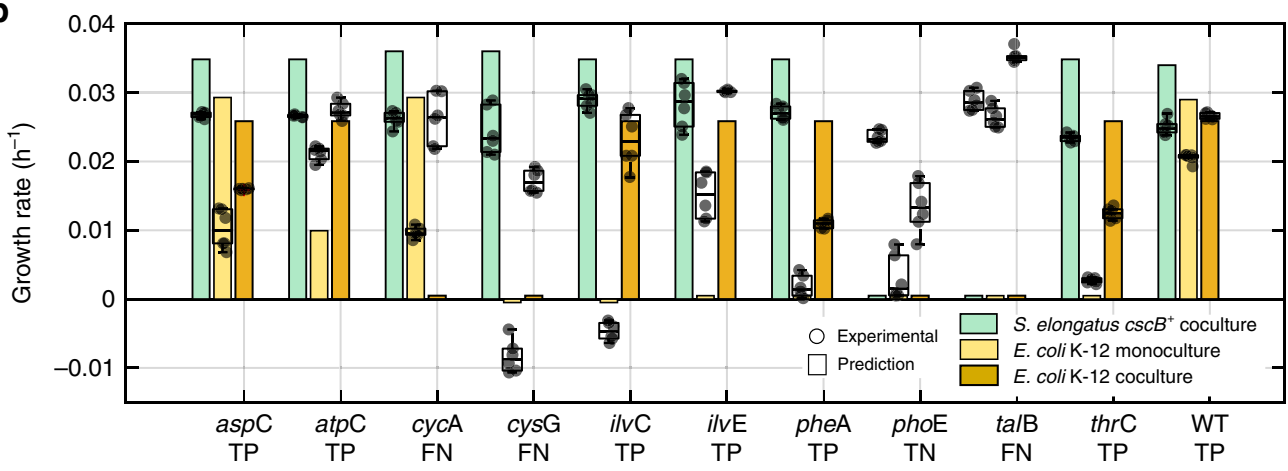

C

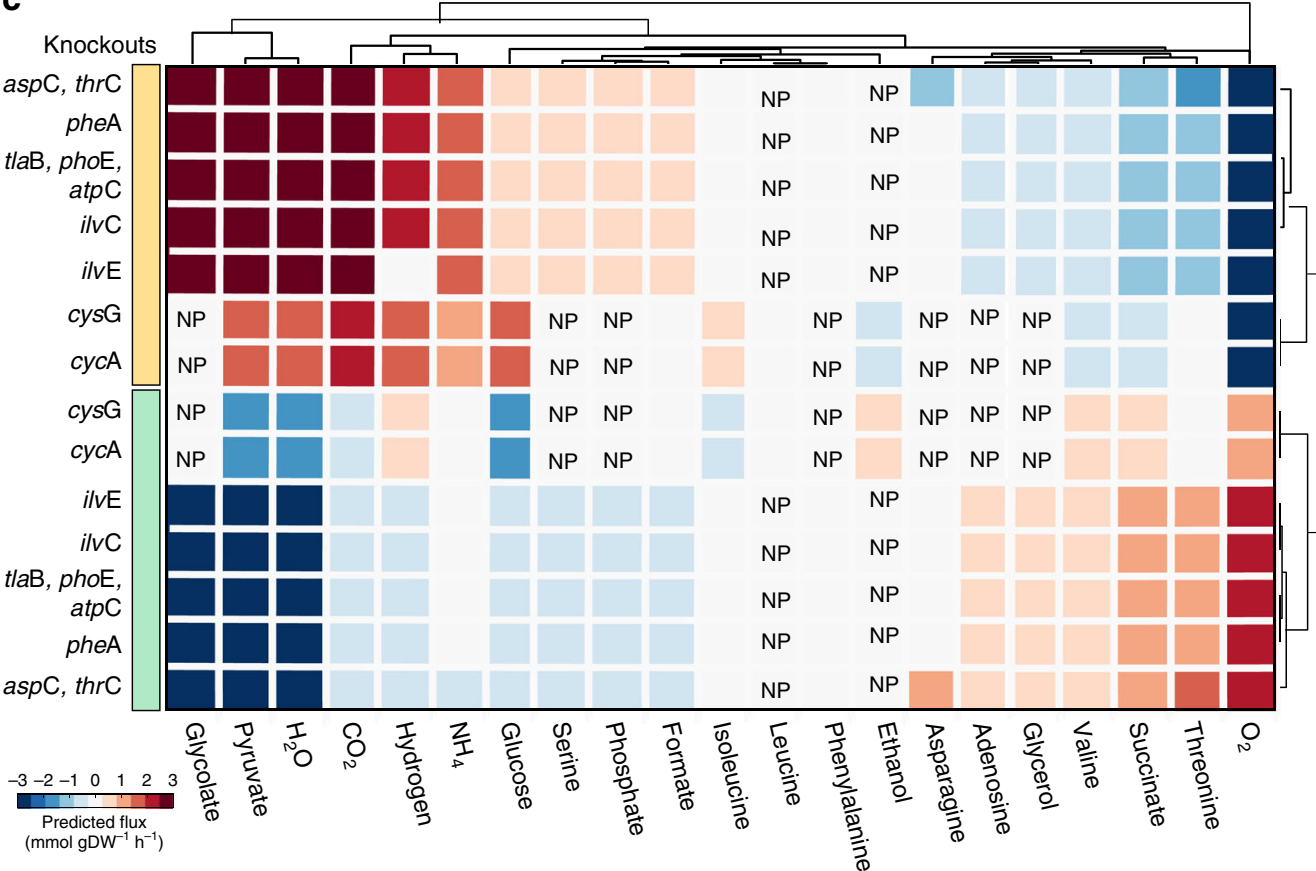

Fig. 4 Community fitness compensates for lethal phenotypic traits by tuning metabolic exchange. a Simulations about resistance to lethal phenotypes across community members. The percentage of knockouts (KOs) with lethal phenotypes was estimated for both community members using the difference of lethal KOs among monoculture and coculture conditions. The percentage reduction is shown for S. elongatus (green) and for the heterotrophs. The communities can reduce their total lethal phenotypic outcomes by 5-9\%. However, the heterotrophic partner benefited disproportionately, reducing the number of lethal traits by at least $7 \%$ for $E$. coli W and up to $27 \%$ for $Y$. lipolytica. The percentage of lethality reduction was determined using the number of genes that are lethal in monoculture vs coculture. The complete dataset is given in Supplementary Data 8, 9. b Experimental validation of selected genes with resistance phenotypes. Six E. coli K-12 mutant strains with lethal phenotypes (ilvC, cysG, ilvE, pheA, phoE, and thrC) and four with nonlethal phenotypes $(\operatorname{aspC}, \operatorname{cyc} \mathrm{A}, \mathrm{tal} \mathrm{B}$, and $\operatorname{atpC})$ were experimentally tested under monoculture and coculture conditions. Predicted growth phenotypes are shown with bars and experimental results with boxplots. In boxplots the central mark indicates the median, and the bottom and top edges of the box indicate the 25th and 75th percentiles, respectively. Benchmarking of the models is achieved by comparing experimental and predicted outcomes as true positive (TP), true negative (TN), false positive (FP), and false negative (FN). c Predicted metabolic exchange shifts in accordance with the KO studied. Complete-linkage clustering shows metabolic exchange associations by genes ( $y$-axis) and metabolites ( $x$-axis). The effect of each KO is shown for $S$. elongatus (green) and $E$. coli K-12 (yellow). Flux distributions were normalized by using the flux distribution obtained under wild-type conditions for both members. Predicted metabolic exchange of $t a / B$, phoE, and $a t p C$ knockouts is represented in one row as the shifts were identical. Similarly, results were found for the metabolic exchange of $\mathrm{KOs}$ aspC and thrC. NP stands for metabolite not predicted to exchange under given condition. Source data underlying Fig. $4 \mathrm{~b}$ are provided as a Source Data file.

have previously been reported in experimental studies. For example, pyruvate and glycerol, which were secreted by our SPCs (Supplementary Figs. 3, 4), were also detected to be secreted by the phototroph Auxenochlorella protothecoides, and incorporated by heterotrophic partners ${ }^{15}$. In turn, the heterotrophs provided vitamin precursors, increasing the growth rate and lipid productivity of the phototroph ${ }^{15}$.

Constraint-based modeling of microbial communities is a promising approach for developing, and controlling robust and stable multi-organismal biotechnological processes. In particular, manually curated CM-models, which compile knowledge about network connectivity, cell communication, and overall function in great detail $22,24,44$. Our CM-model for S. elongatus and E. coli K-12 predicted that activation/deactivation of transport activity associated with the thylakoid and histidine metabolism can potentially relax metabolic bottlenecks. Simulations were validated by transcriptional and metabolomic analyses of the photobiont while cocultivated with E. coli. Both flux predictions and 
expression data showed significant changes in core genes of energy metabolism, carbon fixation, and cofactor and vitamin synthesis. These results are in sync with a previous expression analysis for the SPC of Thermosynechococcus elongatus BP-1 and Meiothermus ruber strain A, in which significant differential expression was observed in phototroph-specific pathways ${ }^{45}$.

Mathematical models of heterotrophic-autotrophic communities have been previously published, encompassing a limited number of parameters, such as inorganic carbon, nitrogen, phosphorous, and light uptake rates ${ }^{46,47}$. However, CM-models provide insight into diverse environmental and genetic stages (here we used 9369 metabolites associated with 11,129 reactions and 7627 genes total), as well as into the interactions among stages for each microbial community. The broader potential of CM-model simulations enables quantitative insights into metabolic exchange and pathway fitness. Other approaches, e.g., one developed for the diatom Phaeodactylum tricornutum and the bacterium Pseudoalteromonas haloplanktis ${ }^{48}$, using a combination of kinetic and constraint-based models, require determination of specific exchange rates (e.g., glutamine, threonine, asparagine, serine and aspartate) for simulation. Experimental quantification of these parameters can be challenging even for monocultures $^{23}$, limiting the simulation capabilities of these multiscale models. The shared metabolite pool provides strong versatility to $\mathrm{CM}$-models to determine production capabilities of each microbial community as well as to track quantitative biochemical fluxes and therefore specific resources used to produce metabolites of interest.

Usually, inhibitory effects can diminish the growth of microorganisms in their natural environment and in many industrial applications ${ }^{49,50}$, but our modeling predictions for all SPCs indicated that cocultivation can help circumvent potential inhibitory effects by redirecting flux and metabolic exchanges between members (Fig. 4). An experimental study of the SPC composed of the diatom $P$. tricornutum and the bacterium Alteromonas macleodii demonstrated that the $\mathrm{KO}$ of nitrate reductase in the heterotrophic partner was compensated by the diatom. This phenotype was attributed to possible metabolic exchange of other nitrogenized compounds ${ }^{51}$. Other studies have also linked nitrate or ammonium availability with a defined type of microbial interaction (e.g., competition, mutualism, or amensalism) $)^{21,22,52}$. Additionally, experimental results using transposon sequencing ( $\mathrm{TN}$-seq) applied to microbial communities have shown differences in gene essentiality between monoand coculture conditions ${ }^{53-55}$. This effect, described as community dependent essentiality, resulted in similar phenotypic outcomes as generated by our metabolic modeling approach. For example, TN-seq data of E. coli collected during cocultivation with Hafnia alvei, Geotrichum candidum, and Penicillium camembertii ${ }^{54}$ showed similar gene essentiality $(a s p \mathrm{C}, i l v \mathrm{E}, i l v \mathrm{C}$, thr C, cor A, pheA, and thrC) as our SPC of S. elongates and E. coli. Interestingly, gene essentially of the ATP synthase/thiamin triphosphate synthase complex (atpC in our SPC) was also observed in a different coculture composed of Staphylococcus aureus and Pseudomonas aeruginosa (atpD) $)^{53}$ hinting at commonalities in the evolution of diverse microbial communities that could be independent of the members. This intriguing hypothesis requires additional experimental confirmation.

To date, a lack of detailed knowledge about microbial communities has limited our ability to predict their responses and redirect their activity for improved bioproduction ${ }^{23,56}$. However, the development of innovative modeling tools that enable quantitative identification of potential metabolic drivers among a myriad of physiological stages (i.e., environmental and genomic) results in solid advances toward a more comprehensive understanding of microbial community structure and function.
Additionally, combinations of these model-driven hypotheses with omic studies will help guide the design and execution of synthetic phototrophic microbial communities.

\section{Methods}

Community-metabolic network reconstruction. CM-models were reconstructed using M-models of S. elongatus PCC7942, iJB792 ${ }^{32}$, B. subtilis, $i \mathrm{YO}^{2} 44^{30}, Y$. lipolytica, $i \mathrm{Yali4}^{31}$, E. coli $\mathrm{W}$ and $\mathrm{K}-12, i \mathrm{ECW} 1372^{28}$ and $i \mathrm{ML} 1514^{29}$. Additional information about the models is shown in Table 1 . All models were subjected to quality control and assessment tests (QC/QA) and integrated as CM-models using the COnstraint-Based Reconstruction and Analysis (COBRA) Toolbox ${ }^{20}$. The COBRA Toolbox is a MATLAB (MathWorks Inc., Natick, MA) software suite for quantitative prediction of cellular and multicellular biochemical networks, implementing the most comprehensive collection modelling methods and algorithms to perform high-throughput model-driven analysis ${ }^{20}$.

M-models were combined through the Shared Metabolite Pool (SMP) as described by Zuniga et al. ${ }^{22}$. This compartment streamlines the connectivity of individual models and the extracellular space based on each microorganism's capabilities. Metabolites suitable for sharing were manually curated based on experimental data of community members in isolation. We performed a highthroughput growth assay (BIOLOG Inc, Hayward, CA) using S. elongatus, testing over 180 different carbon sources (see Supplementary Data 1). Metabolites added to the SMP for E. coli, B. subtillis, and Y. lipolytica were set in accordance with existing data in each M-model and existing information in the literature $29,31,57,58$ We adjusted the $i$ Yali4 metabolic network to represent the genotype of $Y$. lipolytica Polg, in particular the leucine auxotrophy was fulfilled by the exchange of isoleucine. Metabolic models were shared following the standard protocols for computational analysis ${ }^{59}$.

Simulation tools and constraints. CM-models benchmarking was performed using the Gurobi Optimizer Version 5.6.3 solver (Gurobi Optimization Inc., Houston, Texas) in the COBRA Toolbox ${ }^{20}$ for MATLAB. We simulated the maximal growth rate of the community members using the Flux Balance Analysis (FBA)-associated algorithm OptCom ${ }^{20}$. This algorithm maximizes for the biomass objective functions or biomass reactions, which contain all metabolites that comprise organism biomass (e.g., carbohydrates, lipids, and proteins). The growth rate of the population results from the sum of fluxes through both biomass reactions of individual models. Constraints about $\mathrm{CO}_{2}$ and light consumption rates were imported from the $i$ JB792 model. The sucrose secretion rate of $S$. elongatus was constrained to $0.182 \mathrm{mmol} \mathrm{gDW}^{-1} \mathrm{~h}^{-1}$. Benchmarking of experimental and predicted results was performed by calculating modeling statistics for the community members under mono- and coculture: true positive (TP), true negative (TN), false positive (FP), false negative (FN), and the confusion matrix and various measures of quality, such as accuracy, specificity, sensitivity, positive predicted, negative predicted, and Matthews correlation coefficient $(\mathrm{TP}+\mathrm{TN} /(\mathrm{TP}+\mathrm{TN}+\mathrm{FP}+\mathrm{FN}))^{60}$. Metabolites productivity calculations were generated by constraining each metabolite production rate $(0-2$ mmol $\mathrm{gDW}^{-1} \mathrm{~h}^{-1}$ ) and maximizing for the biomass growth of both community members at the same time. We considered metabolite production feasible if both community members grew; these metabolites were selected and are shown in Fig. 2a. Phenotype phase planes were used to estimate the average growth and production rate while synthesizing butanal, ethanol, formaldehyde, and succinate. Growth and production rates under monoculture conditions for engineered strains were processed using the B10NUMB3R5 database ${ }^{61}$. Examples of the calculations are given in Supplementary Data 3.

Robustness analysis and syntrophic pathways. FBA was used to identify metabolic exchange and to perform robustness analysis ${ }^{20}$. Exchange rates of these metabolites were iteratively varied to zero flux or double flux. Simulations deployed growth rate sensitivity of each member in the community. Core sets of vital metabolic exchanges were defined and compared for all CM-models (Supplementary Data 1). The solution space of M-models and CM-networks was scanned by uniformly sampling the solution space using optGpSampler ${ }^{62}$. All models were reduced for sampling. This unbiased assessment provides the possible flux distributions of the network at given constraints ${ }^{63}$. Sampling flux distributions were analyzed using the Symbolic Math Toolbox for MATLAB. The sampling results indicated the metabolic pathways/subsystems activity under monoculture and coculture conditions, and changes in pathway usage due to cocultivation were found.

We developed a method to compare expression data of individual microorganisms and communities with predicted flux distributions.

Transcriptomic data for the pair S. elongatus and E. coli W was collected under monoculture and coculture conditions. Predicted flux distributions were normalized using the growth rate $(\mu)$ of individual microorganisms when using $M$ models and predicted growth rate of the community member when using CMmodels, Eq. (1), where $i$ denotes the index of the reaction and $j$ the microorganism. The number of active reactions by subsystem is denoted by $n$ and the total subsystems in the network by $k$, Eq. (2). For example $S n_{k, j}$ will contain the total normalized flux by subsystem $(k)$ for the community member $(j)$, Eq. (3).

Supplementary Fig. 5 shows $S n_{k, j}$ obtained values for the SPC S. elongatus and E. 
coli $\mathrm{K}-12$.

$$
\begin{gathered}
\text { Flux }_{i, x}=\frac{\text { Flux }_{i, j}}{\mu_{j}} \\
\text { Pathway } \text { flux }_{k, j}=\sum_{i=1}^{i=n} \operatorname{Flux}_{k, j} \\
S n_{k, j}=\frac{\text { Pathway flux }_{k, j}}{\mid \text { Pathway flux }_{k, j} \mid}
\end{gathered}
$$

We explicitly determined the term normalized subsystem $\left(S n_{k, j}\right)$ for all subsystems, conditions, and community members. The culture condition (monoculture, coculture) exits at $m$. Predicted fluxes and gene expression data were normalized to values from -1 to 1 and compared using two sided $t$-test of The Statistics and Machine Learning Toolbox for MATLAB. The flux variability was determined using $\mathrm{FVA}^{64}$.

The difference in $S n_{k, j}$ from mono- to coculture conditions defines pathway usage as syntrophically active (i.e., higher flux), neutrally active (i.e., no change in flux), and deactivated (i.e., decrease in flux). Syntrophically active pathways were assumed to uptake or secrete mass from and to the partner as a result of metabolic exchange. Thus, $S n_{k, j, m}$ represents each pathway and can take values from -1 to 1 depending on the total carried flux under mono- $(\mathrm{m} 1)$ and coculture $(\mathrm{m} 2)$ conditions, Eq. (4) and Eq. (5). The uptake of mass from the SMP to the network was given by:

$$
S n_{k, j, m 1} \in\{0,1\}>S n_{k, j, m 2} \vee S n_{k, j, m 1} \in\{-1,0\}>S n_{k, j, m 2}
$$

And the secretion of metabolites to the SMP by:

$$
S n_{k, j, m 1} \in\{-1,0\}>S n_{k, j, m 2} \vee S n_{k, j, m 1} \in\{0,1\}>S n_{k, j, m 2}
$$

The normalized values by subsystem defined the flux change or flux preservation in each pathway.

Genetic stability depends on the phototrophic community participant. Genetic stability affects members' prevalence and depends on the metabolic interactions between members. CM-models enabled quantification of the resistance to genetic damage in community members by studying metabolic network performance under genetic constraints. Single gene knockout (KO) simulations of over 12,800 genes in the metabolic networks (monoculture and community) were performed in turn, determining growth phenotypes under monoculture and community conditions. Genes predicted to be essential and non-essential were compared across conditions and growth patterns stabilizing, improving or decreasing the growth of the community members were determined.

Strains and culturing conditions. S. elongatus PCC7942 (ATCC \#33912) was engineered to secrete sucrose through the expression of the sucrose/proton symporter $c s c B^{+33}$. Inoculum culture of sucrose-secreting cyanobacterium was prepared using BG-11 (Sigma-Aldrich) medium supplemented with $1 \mathrm{~g} \mathrm{~L}^{-1}$ HEPES (pH 8.9) and $100 \mathrm{mM} \mathrm{NaCl}$ at $35^{\circ} \mathrm{C}$. BG-11 medium is composed of (in $1 \mathrm{~L}$ ): $1.5 \mathrm{~g}$ $\mathrm{NaNO}_{3}, 0.04 \mathrm{~g} \mathrm{~K}_{2} \mathrm{HPO}_{4}, 0.075 \mathrm{~g} \mathrm{MgSO}_{4} \cdot 7 \mathrm{H}_{2} \mathrm{O}, 0.036 \mathrm{~g} \mathrm{CaCl}_{2} \cdot 2 \mathrm{H}_{2} \mathrm{O}, 0.006$ g Citric Acid. $\mathrm{H}_{2} \mathrm{O}, 0.006 \mathrm{~g}$ Ferric Ammonium Citrate, $0.001 \mathrm{~g} \mathrm{Na}_{2}$ EDTA.2 $\mathrm{H}_{2} \mathrm{O}, 0.02 \mathrm{~g}$ $\mathrm{Na}_{2} \mathrm{CO}_{3}, 1 \mathrm{~mL}$ BG-11 trace metals solution. The BG-11 trace metals solution (in $1 \mathrm{~L})$ consists of: $2.86 \mathrm{~g} \mathrm{H}_{3} \mathrm{BO}_{3}, 1.81 \mathrm{~g} \mathrm{MnCl}_{2} \cdot 4 \mathrm{H}_{2} \mathrm{O}, 0.22 \mathrm{~g} \mathrm{ZnSO}_{4} \cdot 7 \mathrm{H}_{2} \mathrm{O}, 0.39 \mathrm{~g}$ $\mathrm{Na}_{2} \mathrm{MoO}_{4} \cdot 2 \mathrm{H}_{2} \mathrm{O}, 0.079 \mathrm{~g} \mathrm{CuSO}_{4} \cdot 5 \mathrm{H}_{2} \mathrm{O}, 49.4 \mathrm{mg} \mathrm{Co}\left(\mathrm{NO}_{3}\right)_{2} \cdot 6 \mathrm{H}_{2} \mathrm{O}$. Cultures were illuminated $24 \mathrm{~h}$ a day while shaking at 150 rounds per minute ${ }^{7,8}$. All experiments were performed with six to eight replicates. Flask cocultures were completed in $25 \mathrm{~mL}$ volumes in baffled flasks. Cultures were plated on rich media to check for contamination. Once cyanobacteria were exposed to coculture media (CoBBG-11 or CoYBG-11) no subsequent subcultures were made to avoid evolution away from sucrose secretion. To prepare precultures for S. elongatus, S. elongatus cultures at exponentially growing phase were centrifuged and washed twice with BG-11 and suspended in a proper volume of coculture medium. To prepare precultures for heterotrophic strains (B. subtilis, E. coli, and Y. lipolytica), single colonies were picked into LB-Miller media and grown until turbid at $37^{\circ} \mathrm{C}$. Cells were diluted into the appropriate coculture media containing $2 \%$ sucrose to acclimate the cells to coculture media, and maintained within log-phase growth $\left(\mathrm{OD}_{600}<0.70\right)$ before use in cocultures. The log-phase cultures were centrifuged and washed twice with BG-11 and suspended in a proper volume of coculture medium. Coculture inoculum was prepared from cultures growing in exponential phase $\left(\mathrm{OD}_{750} 0.5\right)$ using $\sim 2.5 \times 10^{7}$ of $S$. elongatus $\left(0.1 \mathrm{OD}_{750}\right)$ and $\sim 7.5 \times 10^{6}$ of heterotrophs $(0.01$ $\mathrm{OD}_{600}$ ). Experiments were carried out in batch cultures. Samples were obtained in exponential phase assuming pseudo steady state. The Synechococcus elongatus biomass concentration was analyzed by FACSCalibur flow cytometer (BD Biosciences, San Jose, CA) after adding BD Liquid Counting Beads (BD Biosciences, San Jose, CA). The absolute cell numbers in samples were determined by comparing cellular events to the counting beads events measured by the flow cytometer using the equation provided in the kit's total dissolved solids document. For the heterotrophic strains, serial dilutions were plated on LB plates to determine cell numbers. Colony-forming units (CFU) were counted after overnight incubation at $37^{\circ} \mathrm{C}$. All heterotrophs were propagated in BG-11 (Sigma-Aldrich) using the same methods of Hays et al. ${ }^{8}$, while E. coli and B. subtilis 168 strains were maintained in LB-Miller at $37^{\circ} \mathrm{C}$ and Y. lipolytica Polg was maintained in YNB media at $25^{\circ} \mathrm{C}$ as recommended by Yeastern Biotech Co. (Taipei, Taiwan). Strains were streaked from frozen stocks on LB plates before growth in liquid media. Seven $E$. coli knockout strains (asp C, atpC, $c y c \mathrm{~A}, c y s \mathrm{G}$, ilv $\mathrm{C}$, ilvE, phe A, pho E, talB, and $t h r \mathrm{C})$ were obtained from the Keio collection ${ }^{65}$ in Pamela Silver's Lab at Harvard University. Knockout experiments in rich media were performed in 24-well plates using $0.8 \mathrm{~mL}$ of $\mathrm{LB}+0.2 \mathrm{~mL}$ of BG-11 $+0.4 \%$ glucose. Inoculums were prepared as in growth experiments and we kept same culture conditions. Cell numbers were determined using LB agar plates. Samples were collected at $0,8,24,32$, and $48 \mathrm{~h}$. The experiment was performed in triplicates.

Analytical methods. Exchange capabilities of S. elongatus were determined experimentally by performing growth assays on 180 carbon sources. Biolog plates PM1 and PM2 were obtained from BIOLOG Inc. and the manufacturer's protocol was followed ${ }^{66}$. Starting culture density was $\mathrm{OD}_{750}=0.3$, culture media was standard BG11, incubated $25-27^{\circ} \mathrm{C}$. Biolog plates were run in duplicate. The plates were incubated in a light room and examined at $490 \mathrm{~nm}$ for dye absorbance alterations and at $750 \mathrm{~nm}$ to assess optical density. Intracellular metabolites of $E$. coli and S. elongatus were extracted by the methanol-chloroform-water extraction method. This yielded $8.39 \mathrm{mg}$ and $7.46 \mathrm{mg}$ of dry metabolite extract, respectively. One milligram of each extract was used for NMR (Varian Direct Drive (VNMRS) $600 \mathrm{MHz}$ spectrometer, Agilent Technologies, Santa Clara, CA, USA) and GC-MS (Agilent 6890 N, MSD 5975B, Santa Clara, CA, USA) ${ }^{67}$. Medium samples were dried and derivatized as well. 1D ${ }^{1} \mathrm{H}$ NMR analysis was done with normal setting values for global metabolomics ${ }^{68}$. The remaining $7.39 \mathrm{mg}$ and $6.46 \mathrm{mg}$ were dissolved in $180 \mu \mathrm{L} \mathrm{D}_{2} \mathrm{O}$ with $0.5 \mathrm{mM}$ NMR standard for NMR data acquisition.

RNA extraction, library generation and sequencing were performed by harvesting and snap-freezing cells in liquid nitrogen in biological triplicates for each condition. Cell lysates were prepared by grinding the frozen cell pellets in liquid nitrogen with $400 \mu$ l of RLT buffer (Qiagen RNeasy kit). RNA was stabilized by the addition of $2 \mathrm{ml}$ Trizol reagent (Thermo Fisher Scientific) to each $1 \mathrm{ml}$ of lysate. Total RNA was extracted using the RNeasy kit (Qiagen). mRNA was enriched using the Dynabeads mRNA purification kit (Invitrogen). Sequencing libraries were generated using the KAPA RNA HyperPrep kit (Roche) and following the recommended protocol. The libraries were paired-end sequenced on an Illumina HiSeq TM 4000, using 100 bp cycle kits. The sequencing adaptors were trimmed using the trim_galore program ${ }^{69}$. The reads were aligned to the $S$. elongatus genome in the NCBI database (Assembly No. GCF_000012525.1). Subread package-featureCounts (version 1.5.0-p1) ${ }^{70}$ was used to determine reads per each coding region. The aligned sequencing reads were used to determine RNA expression as reads per kilobase per million. The aligned reads were also used to determine differential gene expression using DESeq2 ${ }^{71}$.

Reporting summary. Further information on research design is available in the Nature Research Reporting Summary linked to this article.

\section{Data availability}

Data supporting the findings of this work are available within the paper and its Supplementary Information files. A reporting summary for this Article is available as a Supplementary Information file. The datasets generated and analyzed during the curren study are available from the corresponding author upon request. All sequencing reads were deposited in the Sequence Read Archive under BioProject PRJNA642094, with specific numbers listed in Supplementary Data 6. Additionally, all materials are available at https://github.com/cristalzucsd/SyntheticMicrobialCommunities. Source data are provided with this paper.

\section{Code availability}

Code is available at GitHub [https://github.com/cristalzucsd/

SyntheticMicrobialCommunities].

Received: 22 August 2019; Accepted: 2 July 2020;

Published online: 30 July 2020

\section{References}

1. Stuart, R. K. et al. Cyanobacterial reuse of extracellular organic carbon in microbial mats. ISME J. 10, 1240-1251 (2016)

2. Seneviratne, G. \& Indrasena, I. K. Nitrogen fixation in lichens is important for improved rock weathering. J. Biosci. 31, 639-643 (2006).

3. Bolhuis, H., Cretoiu, M. S. \& Stal, L. J. Molecular ecology of microbial mats FEMS Microbiol. Ecol. 90, 335-350 (2014).

4. Vera, J.-Pde et al. Survival potential and photosynthetic activity of lichens under mars-like conditions: a laboratory study. Astrobiology 10, 215-227 (2010). 
5. Makkonen, S., Hurri, R. S. K. \& Hyvarinen, M. Differential responses of lichen symbionts to enhanced nitrogen and phosphorus availability: an experiment with Cladina stellaris. Ann. Bot. 99, 877-884 (2007).

6. Goff, L. J. Algal Symbiosis: A Continuum of Interaction Strategies (Cambridge University Press, 2011).

7. Li, T. et al. Mimicking lichens: incorporation of yeast strains together with sucrose-secreting cyanobacteria improves survival, growth, ROS removal, and lipid production in a stable mutualistic co-culture production platform. Biotechnol. Biofuels 10, 55 (2017).

8. Hays, S. G., Yan, L. L. W., Silver, P. A. \& Ducat, D. C. Synthetic photosynthetic consortia define interactions leading to robustness and photoproduction. J. Biol. Eng. 11, 4 (2017).

9. Smith, M. J. \& Francis, M. B. A designed A. vinelandii-S. elongatus coculture for chemical photoproduction from air, water, phosphate, and trace metals. ACS Synth. Biol. 5, 955-961 (2016).

10. Smith, M. J. \& Francis, M. B. Improving metabolite production in microbial co-cultures using a spatially constrained hydrogel. Biotechnol. Bioeng. 114, 1195-1200 (2017).

11. Li, T. et al. Creating a synthetic lichen: mutualistic co-culture of fungi and extracellular polysaccharide-secreting cyanobacterium Nostoc PCC 7413. Algal Res. 45, 101755 (2020)

12. Löwe, H., Hobmeier, K., Moos, M., Kremling, A. \& Pflüger-Grau, K. Photoautotrophic production of polyhydroxyalkanoates in a synthetic mixed culture of Synechococcus elongatus $\operatorname{cscB}$ and Pseudomonas putida cscAB. Biotechnol. Biofuels 10, 190 (2017).

13. Grilli, J., Barabás, G., Michalska-Smith, M. J. \& Allesina, S. Higher-order interactions stabilize dynamics in competitive network models. Nature $\mathbf{0}, 1$ (2017).

14. Toledo-Cervantes, A., Madrid-Chirinos, C., Cantera, S., Lebrero, R. \& Muñoz, $\mathrm{R}$. Influence of the gas-liquid flow configuration in the absorption column on photosynthetic biogas upgrading in algal-bacterial photobioreactors. Bioresour. Technol. 225, 336-342 (2017).

15. Higgins, B. T. et al. Cofactor symbiosis for enhanced algal growth, biofuel production, and wastewater treatment. Algal Res. 17, 308-315 (2016).

16. Marín, D. et al. Influence of the seasonal variation of environmental conditions on biogas upgrading in an outdoors pilot scale high rate algal pond. Bioresour. Technol. 255, 354-358 (2018).

17. Buchan, A., LeCleir, G. R., Gulvik, C. A. \& González, J. M. Master recyclers: features and functions of bacteria associated with phytoplankton blooms. Nat. Rev. Microbiol. 12, 686-698 (2014)

18. Liu, J. et al. Advanced nutrient removal from surface water by a consortium of attached microalgae and bacteria: a review. Bioresour. Technol. 241, 1127-1137 (2017).

19. Speirs, J., McGlade, C. \& Slade, R. Uncertainty in the availability of natural resources: fossil fuels, critical metals and biomass. Energy Policy 87, 654-664 (2015).

20. Schellenberger, J. et al. Quantitative prediction of cellular metabolism with constraint-based models: the COBRA Toolbox v2.0. Nat. Protoc. 6, 1290-1307 (2011).

21. Tibocha-Bonilla, J. D., Zuñiga, C., Godoy-Silva, R. D. \& Zengler, K. Advances in metabolic modeling of oleaginous microalgae. Biotechnol. Biofuels 11, 241 (2018).

22. Zuñiga, C. et al. Environmental stimuli drive a transition from cooperation to competition in synthetic phototrophic communities. Nat. Microbiol. 4, 2184-2191 (2019).

23. Zuñiga, C., Zaramela, L. \& Zengler, K. Elucidation of complexity and prediction of interactions in microbial communities. Microb. Biotechnol. 10, 1500-1522 (2017).

24. Pacheco, A. R., Moel, M. \& Segrè, D. Costless metabolic secretions as drivers of interspecies interactions in microbial ecosystems. Nat. Commun. 10, 103 (2019).

25. Zengler, K. \& Palsson, B. O. A road map for the development of community systems (CoSy) biology. Nat. Rev. Microbiol. 10, 366-372 (2012).

26. Tibocha-Bonilla, J. D. et al. Dynamic resource allocation drives growth under nitrogen starvation in eukaryotes. npj Syst. Biol. Appl. 6, 14 (2020).

27. Hays, S. G. \& Ducat, D. C. Engineering cyanobacteria as photosynthetic feedstock factories. Photosynth. Res. 123, 285-295 (2015).

28. Monk, J. M. et al. Genome-scale metabolic reconstructions of multiple Escherichia coli strains highlight strain-specific adaptations to nutritional environments. Proc. Natl Acad. Sci. USA 110, 20338-20343 (2013).

29. Monk, J. M. et al. iML1515, a knowledgebase that computes Escherichia coli traits. Nat. Biotechnol. 35, 904-908 (2017).

30. Oh, Y.-K., Palsson, B. O., Park, S. M., Schilling, C. H. \& Mahadevan, R. Genome-scale reconstruction of metabolic network in Bacillus subtilis based on high-throughput phenotyping and gene essentiality data. J. Biol. Chem. 282, 28791-28799 (2007).
31. Kerkhoven, E. J., Pomraning, K. R., Baker, S. E. \& Nielsen, J. Regulation of amino-acid metabolism controls flux to lipid accumulation in Yarrowia lipolytica. npj Syst. Biol. Appl. 2, 16005 (2016).

32. Broddrick, J. T. et al. Unique attributes of cyanobacterial metabolism revealed by improved genome-scale metabolic modeling and essential gene analysis. Proc. Natl Acad. Sci. USA 113, E8344-E8353 (2016).

33. Ducat, D. C., Avelar-Rivas, J. A., Way, J. C. \& Silver, P. A. Rerouting carbon flux to enhance photosynthetic productivity. Appl. Environ. Microbiol. 78, 2660-2668 (2012)

34. Hong, S.-P. et al. Engineering Yarrowia lipolytica to express secretory invertase with strong FBA1IN promoter. Yeast 29, 59-72 (2012).

35. Lynd, L., Wayman, C., Laser, M., Johnson, D. \& Landucci, R. Strategic biorefinery analysis: analysis of biorefineries. 6-30 (2005).

36. U.S. Environmental Protection Agency. Profile of the organic chemical industry (EPA, 1995).

37. Buschke, N., Schäfer, R., Becker, J. \& Wittmann, C. Metabolic engineering of industrial platform microorganisms for biorefinery applicationsoptimization of substrate spectrum and process robustness by rational and evolutive strategies. Bioresour. Technol. 135, 544-554 (2013).

38. Yang, M. et al. Genetically engineered strains: application and advances for 1,3-propanediol production from glycerol. Food Technol. Biotechnol. 55, 1-15 (2018).

39. García-Pérez, T. et al. Simultaneous methane abatement and PHB production by Methylocystis hirsuta in a novel gas-recycling bubble column bioreactor. Chem. Eng. J. 334, 691-697 (2018).

40. Wakeman, C. A., Goodson, J. R., Zacharia, V. M. \& Winkler, W. C. Assessment of the requirements for magnesium transporters in Bacillus subtilis. J. Bacteriol. 196, 1206-1214 (2014).

41. Ryu, S. \& Trinh, C. T. Understanding functional roles of native pentosespecific transporters for activating dormant pentose metabolism in Yarrowia lipolytica. Appl. Environ. Microbiol. 84, 1-14 (2017).

42. Liu, J. K. et al. Predicting proteome allocation, overflow metabolism, and metal requirements in a model acetogen. PLoS Comput. Biol. 15, e1006848 (2019).

43. Armingol, E., Tobar, E. \& Cabrera, R. Understanding the impact of the cofactor swapping of isocitrate dehydrogenase over the growth phenotype of Escherichia coli on acetate by using constraint-based modeling. PLoS ONE 13, e0196182 (2018).

44. Ponomarova, O. \& Patil, K. R. Metabolic interactions in microbial communities: untangling the Gordian knot. Curr. Opin. Microbiol. 27, 37-44 (2015).

45. Bernstein, H. C. et al. Indirect interspecies regulation: transcriptional and physiological responses of a cyanobacterium to heterotrophic partnership. mSystems 2, e00181-16 (2017).

46. Bordel, S., Guieysse, B. \& Muñoz, R. Mechanistic model for the reclamation of industrial wastewaters using algal-bacterial photobioreactors. Environ. Sci. Technol. 43, 3200-3207 (2009).

47. Solimeno, A. \& García, J. Microalgae-bacteria models evolution: from microalgae steady-state to integrated microalgae-bacteria wastewater treatment models-a comparative review. Sci. Total Environ. 607-608, 1136-1150 (2017)

48. Fondi, M. \& Di Patti, F. A synthetic ecosystem for the multi-level modelling of heterotroph-phototroph metabolic interactions. Ecol. Modell. 399, 13-22 (2019).

49. Lodolo, E. J., Kock, J. L. F., Axcell, B. C. \& Brooks, M. The yeast Saccharomyces cerevisiae -the main character in beer brewing. FEMS Yeast Res. 8, 1018-1036 (2008).

50. Zimmermann, H. F., Anderlei, T., Büchs, J. \& Binder, M. Oxygen limitation is a pitfall during screening for industrial strains. Appl. Microbiol. Biotechnol. 72, 1157-1160 (2006).

51. Diner, R. E., Schwenck, S. M., McCrow, J. P., Zheng, H. \& Allen, A. E. Genetic manipulation of competition for nitrate between heterotrophic bacteria and diatoms. Front. Microbiol. 7, 1-16 (2016).

52. Hom, E. F. Y. \& Murray, A. W. Niche engineering demonstrates a latent capacity for fungal-algal mutualism. Science 345, 94-98 (2014).

53. Ibberson, C. B. et al. Co-infecting microorganisms dramatically alter pathogen gene essentiality during polymicrobial infection. Nat. Microbiol. 2, 17079 (2017).

54. Morin, M., Pierce, E. C. \& Dutton, R. J. Changes in the genetic requirements for microbial interactions with increasing community complexity. elife 7, 1-16 (2018).

55. Lewin, G. R., Stacy, A., Michie, K. L., Lamont, R. J. \& Whiteley, M. Large-scale identification of pathogen essential genes during coinfection with sympatric and allopatric microbes. Proc. Natl Acad. Sci. USA 116, 19685-19694 (2019).

56. Tan, J., Zuniga, C. \& Zengler, K. Unraveling interactions in microbial communities-from co-cultures to microbiomes. J. Microbiol. 53, 295-305 (2015). 
57. Wei, S., Jian, X., Chen, J., Zhang, C. \& Hua, Q. Reconstruction of genomescale metabolic model of Yarrowia lipolytica and its application in overproduction of triacylglycerol. Bioresour. Bioprocess 4, 51 (2017).

58. Henry, C. S., Zinner, J. F., Cohoon, M. P. \& Stevens, R. L. iBsu1103: a new genome-scale metabolic model of Bacillus subtilis based on SEED annotations. Genome Biol. 10, R69 (2009).

59. Rule, A. et al. Ten simple rules for writing and sharing computational analyses. PLoS Comput. Biol. 15, e1007007 (2019).

60. Matthews, B. W. Comparison of the predicted and observed secondary structure of T4 phage lysozyme. BBA Protein Struct. 405, 442-451 (1975).

61. Milo, R., Jorgensen, P., Moran, U., Weber, G. \& Springer, M. BioNumbersthe database of key numbers in molecular and cell biology. Nucleic Acids Res. 38, D750-D753 (2010).

62. Megchelenbrink, W., Huynen, M. \& Marchiori, E. optGpSampler: an improved tool for uniformly sampling the solution-space of genome-scale metabolic networks. PLoS ONE 9, e86587 (2014).

63. Zuñiga, C. et al. Predicting dynamic metabolic demands in the photosynthetic eukaryote Chlorella vulgaris. Plant Physiol. 176, 450-462 (2018).

64. Mahadevan, R. \& Schilling, C. H. The effects of alternate optimal solutions in constraint-based genome-scale metabolic models. Metab. Eng. 5, 264-276 (2003).

65. Baba, T. et al. Construction of Escherichia coli K-12 in-frame, single-gene knockout mutants: the Keio collection. Mol. Syst. Biol. 2, 2006-0008 (2006)

66. Zuñiga, C. et al. Genome-scale metabolic model for the green alga Chlorella vulgaris UTEX 395 accurately predicts phenotypes under autotrophic, heterotrophic, and mixotrophic growth conditions. Plant Physiol. 172, 589-602 (2016).

67. Kim, Y.-M. \& Heyman, H. M. in Methods in Molecular Biology (Humana Press, New York, 2018) pp. 107-118.

68. Weljie, A. M., Newton, J., Mercier, P., Carlson, E. \& Slupsky, C. M. Targeted profiling: quantitative analysis of ${ }^{1} \mathrm{H}$ NMR metabolomics data. Anal. Chem. 78, 4430-4442 (2006).

69. Krueger, F. Trim Galore!: A wrapper tool around Cutadapt and FastQC to consistently apply quality and adapter trimming to FastQ files. https://www. bioinformatics.babraham.ac.uk/projects/trim_galore/ (2015).

70. Liao, Y., Smyth, G. K. \& Shi, W. featureCounts: an efficient general purpose program for assigning sequence reads to genomic features. Bioinformatics $\mathbf{3 0}$, 923-930 (2014).

71. Love, M. I., Huber, W. \& Anders, S. Moderated estimation of fold change and dispersion for RNA-seq data with DESeq2. Genome Biol. 15, 550 (2014).

\section{Acknowledgements}

We acknowledge Stephanie Hays at Pamela Silver's Lab at Harvard Medical School for providing coculture strains and performing pilot experiments. We also thank Fanni Davrazou at The National Renewable Energy Laboratory for providing engineered $Y$. lipolytica strains. Furthermore, we acknowledge Daniel Zielinski at UCSD for providing input throughout all stages of this work and to Clarisse Marotz for proofreading the manuscript. This material is based upon work supported by the National Science Foundation under Grant No. 1332344 and CBET-1804187, and the U.S. Department of Energy (DOE), Office of Science, Office of Biological \& Environmental Research under Awards DE-SC0012658 and DE-SC0019388. C.Z. was in part supported by Mexican National Research Council, CONACYT, fellowship No. 237897.

\section{Author contributions}

C.Z., M.J.B., and K.Z. conceived the study. C.Z. developed CM-models and computational methods as well as performed simulations and experimental design. T.L., J.P.J., and M.G. performed physiological and RNA-seq experiments. K.B. and Y-M.K. performed metabolomics analysis. C.Z., P.B., J.P.J., C-T.L., and M.J.B. discussed the results. C.Z. analyzed the data. C.Z. wrote the manuscript with input from all co-authors.

\section{Competing interests}

The authors declare no competing interests.

\section{Additional information}

Supplementary information is available for this paper at https://doi.org/10.1038/s41467020-17612-8.

Correspondence and requests for materials should be addressed to K.Z.

Peer review information Nature Communications thanks Daniel Ducat, and the other, anonymous, reviewer(s) for their contribution to the peer review of this work. Peer reviewer reports are available.

Reprints and permission information is available at http://www.nature.com/reprints

Publisher's note Springer Nature remains neutral with regard to jurisdictional claims in published maps and institutional affiliations.

Open Access This article is licensed under a Creative Commons Attribution 4.0 International License, which permits use, sharing, adaptation, distribution and reproduction in any medium or format, as long as you give appropriate credit to the original author(s) and the source, provide a link to the Creative Commons license, and indicate if changes were made. The images or other third party material in this article are included in the article's Creative Commons license, unless indicated otherwise in a credit line to the material. If material is not included in the article's Creative Commons license and your intended use is not permitted by statutory regulation or exceeds the permitted use, you will need to obtain permission directly from the copyright holder. To view a copy of this license, visit http://creativecommons.org/ licenses/by/4.0/.

(C) The Author(s) 2020 